\title{
LINEAR OPERATORS AND VECTOR MEASURES
}

\author{
BY \\ J. K. BROOKS(1) AND P. W. LEWIS(2)
}

\begin{abstract}
Compact and weakly compact operators on function spaces are studied. Those operators are characterized by properties of finitely additive set functions whose existence is guaranteed by Riesz representation theorems.
\end{abstract}

1. Introduction. In this paper we study operators on function spaces. Criteria for weak and strong compactness of these operators are established in terms of their representing vector measures. Some of these results have been announced by the authors in [10]. The function spaces are as follows. Let $E$ and $F$ be locally convex spaces, and let $H$ be a locally compact Hausdorff space. By $C_{0}(H, E)$ we denote the space of continuous $E$-valued functions which vanish at $\infty$, by $C(H, E)$ we denote the space of continuous $E$-valued functions equipped with the compact-open topology, and by $U_{E}(\mathfrak{D})$ we denote the space of totally $D$ measurable functions. In $\$ 2$ a Riesz representation theorem for operators $L$ defined on the continuous function spaces into $F$ is given in terms of a measure $m$ on $\Sigma$, the Borel subsets of $H$, with values in $B\left(E, F^{* *}\right)$, where the latter is the space of operators from $E$ to $F^{* *}$ and $E$ and $F$ are locally convex spaces. In symbols, $L(f)=\int f d m$, and we write $L \leftrightarrow m$. Our theorem extends and unifies existing representation theorems of this type. Although a number of authors have considered this problem in special cases, none have used the device of embedding isometrically the simple functions in $C(H, E)^{* *}$ and thus reducing the problem to utilizing the representation theorem for operators $L: U_{E}(\mathcal{D}) \rightarrow F$, which can be easily established.

In order to study weak compactness of operators, when $E$ and $F$ are Banach spaces, one examines the adjoint which maps $F^{*}$ into ca $\left(\Sigma, E^{*}\right)$, the Banach space of $E^{*}$-valued measures of bounded variation. Consequently, the problem is reduced to considering weakly compact subsets of $\mathrm{ca}\left(\Sigma, E^{*}\right)$. This enables us to use recent results of Brooks [6] giving necessary and sufficient conditions for sets to be weakly compact in the space of vector measures. This theory is presented in $\$ 3$ and applied to operators in $\$ 4$. Essentially the criterion for an operator $L \leftrightarrow m$ to be weakly compact is that $\tilde{m}\left(E_{i}\right) \rightarrow 0$ on disjoint sets $E_{i}$, where $\tilde{m}$ is the semivariation of $m$. Other topics in $\$ 4$ include a discussion concerning the problem of when $m$ takes its values in $B(E, F)$ and criteria for operators to be compact in terms of topologies induced on $F_{1}^{*}$ by $m$. In $\$ 5$ various topics are

Received by the editors March 9, 1971 and, in revised form, October 11, 1972.

AMS (MOS) subject classifications (1970). Primary 47B99; Secondary 46G10.

(1) This author was partially supported by National Science Foundation Grant GP-28617.

(2) This author partially supported by a faculty research grant at North Texas State University. Copyright $\odot$ 1974, American Mathematical Society 
discussed; a modified example of Lewis [22] is given which settles questions on limits of strongly bounded vector measures.

2. Representation theorems. In this section we establish integral representation theorems for various function spaces. Numerous authors have worked on this problem. For example, see Bartle, Dunford, and Schwartz [2], Dinculeanu [11], Foiaş and Singer [15], Singer [31], Swong [32], and Tucker [34]. However, none of these authors has made use of the isometry in Lemma 2.1 and of Theorem 2.0. The approach presented here unifies much of the work in this area and shows the applicability of the representation theorem on the space of totally measurable functions and this isometry.

We next establish some notation and terminology. We denote the classes of all continuous seminorms on the locally convex spaces (lcs) $E$ and $F$ by $\{p\}_{E}$ and $\{p\}_{F}$, respectively; if $X$ is an lcs, then $X^{*}$ will be the continuous dual of $X$. If $X$ is a Banach space ( $B$-space), then we view $X$ as a closed linear subspace of $X^{* *}$. The term operator will always be used to refer to a continuous linear transformation, and the term measure will be used to refer to a finitely additive set function. The reader should recall that a bounded regular complex valued finitely additive set function defined on an algebra $\mathfrak{D}$ of subsets of a compact space $S$ is countably additive, e.g. see [13, p. 138]. We denote the characteristic function of a set $A$ by $\mathfrak{X}_{A}$. The symbol $\mathrm{B}$ will denote $C_{0}(H, E), \mathbf{B}_{c}$ will denote those functions in $C_{0}(H, E)$ with compact support, and $\mathbf{C}$ will denote the space of all continuous $E$ valued functions on $H$; the analogous spaces of scalar valued functions will be denoted by $C_{0}(H), C_{c}(H)$, and $C(H)$. The topology on $\mathrm{B}$ and $\mathrm{B}_{c}$ will be given by uniform convergence, and the topology on $\mathbf{C}$ will be the compact-open topology.

Definition 2.1. Let $\mathfrak{D}$ denote a ring of subsets of a universal space $S$, and let $S_{E}(\mathfrak{D})$ be the collection of all $E$-valued simple functions over the ring $\mathscr{D}$; let $U_{E}(\mathfrak{D})$ be the collection of all $E$-valued functions $f$ which vanish outside of some set in $\mathfrak{D}$ such that $f$ is the uniform limit of a net $\left(f_{\alpha}\right) \subset S_{E}(\mathfrak{D})$. The space $U_{E}(\mathfrak{D})$ is called the space of totally measurable functions; $U_{E}(\mathfrak{D})$ is equipped with the uniform topology.

Definition 2.2. If $L: U_{E}(\mathfrak{D}) \rightarrow F$ is a linear mapping, $A \in \mathfrak{D}, p \in\{p\}_{E}$, and $q \in\{p\}_{F}$ then let

$$
\left\|L_{A}\right\|_{(p, q)}=\sup \left\{q(L(f)): f \in U_{E}(\mathfrak{D}), \mathfrak{X}_{A} \cdot f=f, p(f) \leq 1\right\},
$$

where $p(f)=\sup \{p(f(t)): t \in H\}$. Let $\&$ be the set of linear mappings $L: U_{E}(\mathfrak{D}) \rightarrow F$ so that if $q \in\{p\}_{F}$ then there is a $p \in\{p\}_{E}$ so that $\left\|L_{A}\right\|_{(p, q)}<\infty$ for each $A \in \mathfrak{D}$.

Definition 2.3. If $X$ and $Y$ are arbitrary lcs with the family of all continuous seminorms $\{p\}_{X}$ and $\{p\}_{Y}$ respectively, $m: \mathfrak{D} \rightarrow B(X, Y)$ is finitely additive, $q \in\{p\}_{Y}$, and $A \in \mathfrak{D}$, then define $\tilde{m}_{(p, q)}(A)$ to be

$$
\sup _{g(A), x_{i}}\left\{q\left(\sum_{A_{i} \in \pi(A)} m\left(A_{i}\right) \cdot x_{i}\right)\right\}
$$


where $\pi(A)$ denotes the (disjoint) $D$-partitions of $A$, and $p\left(x_{i}\right) \leq 1$ for each $i$, $p \in\{p\}_{X}$. If $B \subset S$, define $\tilde{m}_{(p, q)}(B)$ to be $\sup \left\{\tilde{m}_{(p, q)}(A): A \subset B, A \in \mathfrak{D}\right\}$.

In the sequel, $\tilde{m}_{(p, q)}$ will be called the $(p, q)$-semivariation, where $(p, q)$ is a pairing determined as in Definition 2.2.

We mention that the bilinear integration theory used here is developed in Dinculeanu [11]. In particular, if $m: \mathfrak{D} \rightarrow B(E, F)$ has finite $(p, q)$-semivariation and $f \in U_{E}(\mathfrak{D})$, then let $\left(f_{\alpha}\right)$ be a net in $S_{E}(\mathfrak{D})$ which converges uniformly to $f$, and define $\int f d m$ to be $\lim \int f_{\alpha} d m$, where the convergence is in the locally convex completion of $F$.

We state the following theorem for reference purposes; it will be referred to as the RRT.

Riesz Representation Theorem [29, p. 131]. To each bounded linear functional $L$ on $C_{0}(X)$, where $X$ is a locally compact Hausdorff space, there corresponds a unique complex regular Borel measure $\mu$ such that

$$
L(f)=\int_{X} f d \mu, \quad f \in C_{0}(X) .
$$

Moreover, if $L$ and $\mu$ are related as in (II.1), then

$$
\|L\|=|\mu|(X) \quad(|\mu| \text { is the total variation of } \mu) .
$$

Theorem 2.0 [11, p. 145]. If $L \in \mathbb{E}$, then there is a unique finitely additive, operator-valued set function $m: \mathfrak{D} \rightarrow B(E, F)$ so that

$$
L(f)=\int_{H} f d m, \quad f \in U_{E}(\mathfrak{D}) .
$$

Furthermore, for each $q \in\{p\}_{F}$ there is a $p \in\{p\}_{E}$ so that $\tilde{m}_{(p, q)}(A)<\infty$ for each $A \in \mathfrak{D}$, and $\tilde{m}_{(p, q)}(A)=\left\|L_{A}\right\|_{(p, q)}$.

Before proceeding further, it will be necessary to make explicit the various topologies we shall be using. If $F$ is an lcs, then the topology on $F^{*}$ will be the strong topology and the topology on $F^{* *}$ will be the $\varepsilon^{00}$-topology. Therefore the topology on $F^{* *}$ will be uniform convergence on the polars of the $p$-unit balls of $F$. If $A$ is an lcs and $p$ is a continuous seminorm on $A$, then $A(p, 1)$ will be the $p$ unit ball of $A$; and if $f^{* *} \in A^{* *}$, then

$$
a_{p}\left(f^{* *}\right)=\sup \left\{\left|f^{* *}\left(f^{*}\right)\right|: f^{*} \in A^{0}(p, 1)\right\},
$$

where $A^{0}(p, 1)$ denotes the polar of $A(p, 1)$.

If $\mathfrak{X}$ is a $B$-space and $r>0$, then $\mathfrak{X}_{r}$ will denote the closed ball of radius $r$ about the origin.

Definition 2.4. Let $\mathcal{L}$ be the subring of $\Sigma$ consisting of those sets whose characteristic functions are pointwise limits of sequences in $C_{c}(H)$. If $A \in \mathcal{L}$ and $\left(\varphi_{n}^{A}\right)$ is a sequence converging pointwise to $\mathfrak{X}_{A}$ so that $0 \leq \varphi_{n}^{A} \leq 1$ for each $n$, 
then $\left(\varphi_{n}^{A}\right)$ is called a determining sequence for $A$. We write $\varphi \prec A$ to denote that $\operatorname{supp}(\varphi) \subset A$.

Definition 2.5 (see [16] and [13, VI.7.2]). If $A \in \Sigma$ and $x \in E$, let $\mathfrak{X}_{A} \cdot x$ be defined on $\mathrm{B}^{*}$ by

$$
\mathfrak{X}_{A} \cdot x\left(f^{*}\right)=\mu_{\left(x, f^{*}\right)}(A), \quad f^{*} \in \mathrm{B}^{*},
$$

where $\mu_{\left(x, f^{*}\right)}$ is the unique regular Borel measure in the $R R T$ given by

$$
\left\langle x, f^{*}\right\rangle(f)=f^{*}(f \cdot x)=\int_{H} f d \mu_{\left(x, f^{*}\right)}=\mu_{\left(x, f^{*}\right)}(f) .
$$

It is clear that $S_{E}(\Sigma)$ is a linear manifold in the lcs of bounded $E$-valued functions defined on $H$; from Definition 2.5, it follows that $S_{E}(\Sigma)$ may be viewed as a subspace of $B^{* *}$. However, if $E$ and $F$ are $B$-spaces, much more can be said, as the following lemma indicates. We give the proof in some detail since we shall have occasion to refer to the construction later.

Lemma 2.1. If $E$ and $F$ are $B$-spaces and

$$
\zeta=\sum_{i=1}^{k} \mathfrak{X}_{A_{i}} \cdot x_{i} \in S_{E}(\Sigma),
$$

then $\|\zeta\|_{B^{* *}}=\|\zeta\|_{\infty}$, where $\|\cdot\|_{\infty}$ denotes the uniform norm.

Proof. Without loss of generality, we may suppose that $\zeta=\sum_{i=1}^{k} \mathfrak{X}_{A_{i}} \cdot x_{i}$ is in the canonical form of a simple function and that $\left\|x_{1}\right\|=\|\zeta\|_{\infty}$. Let $t \in A_{1}$ and define $\xi: \Sigma \rightarrow E^{*}$ in the following way:

(i) $\xi(A)=0$ if $t \notin A$;

(ii) if $t \in A$, then $\xi(A)=x^{*} \in E^{*}$, where $\left\|x^{*}\right\|=1$ and $x^{*}\left(x_{1}\right)=\left\|x_{1}\right\|$.

Then clearly $\xi$ is countably additive, has finite semivariation, and $|\xi|$ (the total variation function) is regular. Furthermore, $\xi \in \mathrm{B}^{*}$, and $\|\xi\|_{B^{*}}=\tilde{\xi}(H)=1$. Now

$$
\begin{aligned}
\zeta(\xi) & =\left(\sum_{i=1}^{k} \mathfrak{X}_{A_{i}} \cdot x_{i}\right)(\xi)=\sum_{i=1}^{k} \mathfrak{X}_{A_{i}} \cdot x_{i}(\xi) \\
& =\sum_{i=1}^{k} \mu_{\left(x_{i}, \xi\right)}\left(A_{i}\right) .
\end{aligned}
$$

Let $i>1, \varepsilon>0$, and choose a collection $\left\{K_{i}\right\}_{i=2}^{k}$ of compact sets so that $K_{i} \subset A_{i}$ and $\sum_{i=2}^{k}|\mu|_{\left(x_{i}, \xi\right)}\left(A_{i}-K_{i}\right)<\varepsilon$. Then let $\left\{V_{i}\right\}$ be a disjoint collection of conditionally compact open sets so that $t \notin U V_{i}$, and $\sum_{i=2}^{k}|\mu|_{\left(x_{i}, \xi\right)}\left(V_{i}-K_{i}\right)<\varepsilon$. By the Baire approximation theorem [19, p. 218], and the Lebesgue dominated convergence theorem, we can choose a collection of compact $G_{8}$ subsets $\left\{G_{i}\right\}$ and $\left\{\varphi_{i}\right\} \subset C(H)$ so that $K_{i} \subset G_{i} \subset V_{i}, \quad 0 \leq \varphi_{i} \leq 1, \quad \operatorname{supp}\left(\varphi_{i}\right) \subset V_{i}$, and $\sum_{i=2}^{k}\left|\mu_{\left(x_{i}, \xi\right)}\left(G_{i}\right)-\mu_{\left(x_{i}, \xi\right)}\left(\varphi_{i}\right)\right|<\varepsilon$. But $\mu_{\left(x_{i}, \xi\right)}(\varphi)=\int \varphi_{i} x_{i} d \xi=0$. Since 


$$
\left(X_{A_{1}} \cdot x_{1}\right)(\xi)=\xi\left(A_{1}\right) \cdot x_{1}=\left\|x_{1}\right\|
$$

it follows that $\|\xi\|_{b_{0}} \leq\|\xi\|_{\text {B... }}$.

For the reverse inequality, let $\zeta$ be as above, and choose $\xi$ arbitrarily in $B_{1}^{*}$. Let $\left\{K_{i}\right\},\left\{G_{i}\right\},\left\{V_{i}\right\}$, and $\left\{\varphi_{i}\right\}$ be chosen as in the preceding paragraph, except that we now insist that $i$ range from 1 to $k$. Therefore

$$
\begin{aligned}
\left|\sum_{i=1}^{k}\left(\mu_{\left(x_{i}, \xi\right)}\left(A_{i}\right)-\mu_{\left(x_{i}, \xi\right)}\left(\varphi_{i}\right)\right)\right| \leq & \left|\sum_{i=1}^{k}\left(\mu_{\left(x_{i}, \xi\right)}\left(A_{i}\right)-\mu_{\left(x_{i}, \xi\right)}\left(G_{i}\right)\right)\right| \\
& +\left|\sum_{i=1}^{k}\left(\mu_{\left(x_{i}, \xi\right)}\left(G_{i}\right)-\mu_{\left(x_{i}, \xi\right)}\left(\varphi_{i}\right)\right)\right|<3 \varepsilon,
\end{aligned}
$$

and

$$
\left|\sum \mu_{\left(x_{i}, \xi\right)}\left(\varphi_{i}\right)\right|=\left|\xi\left(\sum_{i=1}^{k} \varphi_{i} \cdot x_{i}\right)\right| \leq\left\|\sum_{i=1}^{k} \varphi_{i} \cdot x_{i}\right\| \leq\left\|x_{1}\right\| .
$$

This proves the lemma.

Remark 2.1. If $E$ is an lcs, $q \in\{p\}_{E}$, and $\xi=\sum_{i=1}^{k} \mathfrak{X}_{D_{i}} \cdot x_{i} \in S_{E}(\Sigma)$, then from the technique of Lemma 2.1 it follows that

$$
a_{q}\left(\sum_{i=1}^{k} \mathfrak{X}_{D_{i}} \cdot x_{i}\right) \leq q\left(\sum_{i=1}^{k} \mathfrak{X}_{D_{i}} \cdot x_{i}\right) .
$$

Therefore it follows that the second adjoint $L^{* *}: S_{E}(\Sigma) \rightarrow F^{* *}$ is continuous relative to the uniform topology on $S_{E}(\Sigma)$.

The following theorem is used in establishing the representation theorem.

Theorem 2.1. Let $f \in C_{0}(H, E)$. Then there is a net $\left(g_{\alpha}\right) \subset S_{E}(\mathcal{L})$ simultaneously approximating $f$ in the uniform and $\mathrm{B}^{* *}$ topologies.

Proof. For each $p \in\{p\}_{E}$, let $A_{(p, n)}=\{x: p(f(x)) \geq 1 / n\}$. Then $A_{(p, n)} \in \mathcal{L}$ for each $p$ and each $n$ since $A_{(p, n)}$ is a compact $G_{8}$. Let $\mathbf{N}$ denote the natural numbers, and let $\Gamma=\{p\}_{E} \times \mathbf{N}$. For $(p, n),(q, m) \in \Gamma$, define $(p, n) \geq(q, m)$ if and only $p \geq q$ in the ordering on $\{p\}_{E}$ and $n \geq m$. Then $\Gamma$ is a directed set, and for each $\gamma \in \Gamma, \gamma=(p, n)$, let $f_{\gamma}=\mathfrak{X}_{A_{(p, N)}} \cdot f$ be defined as in Definition 2.5. Thus $f_{\gamma} \in \mathbf{B}^{* *}$. It is clear that $f_{\gamma} \rightarrow f$ uniformly. But also $f_{\gamma} \rightarrow f$ in the $\mathbf{B}^{* *}$ topology. In fact, if $x^{*} \in \mathrm{B}^{0}(p, 1)$ and $\varepsilon>0$, then choose $k \in \mathrm{N}$ and let $\gamma \in \Gamma, \gamma=(q, t)$ so that $1 / k \leq \varepsilon / 2$ and $q \geq p, t \geq k$. Using the fact that there is a determining sequence for $A(q, t)$ which is identically one on $A(q, t)$, we see that $\mid f_{y}\left(x^{*}\right)$ $-x^{*}(f) \mid \leq 2 / t<\varepsilon$.

Now let $\lambda=(p, n)$ be a fixed element of $\Gamma$, and note that $f(A(\lambda))$ is a compact subset of $E$. Therefore for each $m \in \mathbf{N}$ there is a partition $\left\{A_{i}^{m}\right\}_{i=1}^{k(m)}$ of $A(\lambda)$ into elements of $\mathfrak{L}$ so that $p$-diam $\left(f\left(A_{i}^{m}\right)\right)<1 / m$ for each $i$. Then, for each $m>n$, choose one such partition $\left\{A_{i}^{m}\right\}_{i=1}^{k(m)}$, let $x_{i}^{m} \in f\left(A_{i}^{m}\right)$, and let 


$$
\zeta(\lambda, m)=\sum_{i=1}^{k(m)} \mathfrak{X}_{A T} \cdot x_{i}^{m}
$$

To simplify notation, we shall usually denote $\zeta(\lambda, m)$ by $\sum_{i=1}^{k(m)} \mathfrak{X}_{A_{i}} \cdot x_{i}$. Order the set $I=\{(\lambda, m): \lambda=(p, n) \in \Gamma, m \geq n\}$ by $(\lambda, m) \geq(\gamma, j)$ if $\lambda \geq \gamma$ and $m \geq j$. It is easy to see that $\{\zeta(\lambda, m)\}_{I} \rightarrow f$ uniformly.

Finally, we prove that $\{\xi(\lambda, n)\}_{I}$ is a net which satisfies the conclusion of the theorem. To this end, let $q \in\{p\}_{E}, \varepsilon>0$, and choose $p \geq q, 1 / n<\varepsilon$, and $\lambda=(d, t) \geq(p, n)$. If $\xi(\lambda, m)=\sum_{i=1}^{k(m)} \mathfrak{X}_{D_{i}} \cdot x_{i}$ is chosen as above and $f^{*} \in$ $\mathbf{B}^{0}(q, 1)$, then

$$
\left|\left(\xi(\lambda, m)-f_{\lambda}\right) f^{*}\right|=\left|\sum_{i=1}^{k(m)}\left(\mu_{\left(x_{i}, f^{*}\right)}\left(D_{i}\right)-\mu_{\left(f, f^{*}\right)}\left(D_{i}\right)\right)\right|,
$$

where $\mu_{\left(x_{i}, f^{*}\right)}$ and $\mu_{\left(f, f^{*}\right)}$ are the regular Borel measures of Definition 2.5. Repeat the construction of Lemma 2.1 to select disjoint open sets $\left\{V_{i}\right\}_{i=1}^{k(m)}$ and compact $G_{\delta}$ sets $\left\{G_{i}\right\}_{i=1}^{k(m)}$ so that $G_{i} \subset V_{i}$ for each $i, d-\operatorname{diam}\left(F\left(V_{i}\right)\right)<2 / t$,

$$
\sum_{i=1}^{k(m)}|\mu|_{\left(x_{i}, f^{*}\right)}\left(G_{i} \Delta D_{i}\right)<\varepsilon
$$

and

$$
\sum_{i=1}^{k(m)}|\mu|_{\left(f, f^{*}\right)}\left(G_{i} \Delta D_{i}\right)<\varepsilon
$$

Therefore

$$
\left|\xi(\lambda, m)\left(f^{*}\right)-f_{\lambda}\left(f^{*}\right)\right|<\left|\sum_{i=1}^{k(m)}\left(\mu_{\left(x_{i}, f^{*}\right)}\left(G_{i}\right)-\mu_{\left(f, f^{*}\right)}\left(G_{i}\right)\right)\right|+2 \varepsilon .
$$

We may apply the Lebesgue dominated convergence theorem since each $G_{i} \in \mathcal{L}$ Thus since $d \geq q$ and $f^{*} \in \mathbf{B}^{0}(q, 1)$,

$$
\left|\sum_{i=1}^{k(m)}\left(\mu_{\left(x_{i}, f^{*}\right)}\left(G_{i}\right)-\mu_{\left(f, f^{*}\right)}\left(G_{i}\right)\right)\right| \leq \frac{4}{t}
$$

whence $\left|\zeta(\lambda, m)\left(f^{*}\right)-f_{\lambda}\left(f^{*}\right)\right| \leq 6 \varepsilon$, and the proof is completed.

Definition 2.6. If $m: \Sigma \rightarrow B\left(E, F^{* *}\right)$ is finitely additive with finite $(p, q)$ semivariation, then we say that $m$ is weakly regular provided that $x \in E$ and $z \in F^{*}$ imply that $m_{(x, z)}(\cdot) \equiv\langle m(\cdot) x, z\rangle$ is a finite regular Borel measure.

We should note here that this notion of weak regularity differs from that in [13].

Theorem 2.2 (Representation Theorem). If $L: B \rightarrow F$ is an operator, then there is a unique weakly regular set function $m: \Sigma \rightarrow B\left(E, F^{* *}\right)$ so that

$$
L(f)=\int_{H} f d m, \quad f \in \mathbf{B} .
$$


Proof. We know that $S_{E}(\Sigma) \subset \mathrm{B}^{* *}$, and therefore $L^{* *}: S_{E}(\Sigma) \rightarrow F^{* *}$. From Corollary 2.1.1, it follows that $L^{* *}$ has a continuous extension $L: U_{E}(\Sigma)$ $\rightarrow C\left(F^{* *}\right)$, where $C\left(F^{* *}\right)$ denotes the locally convex completion of $F^{* *}$. But by Theorem 2.1 and Lemma 2.1 it follows that $L(f)=L^{* *}(f)=L(f)$. Now we apply Theorem 2.0 and write $L(f)=\int_{H} f d m$. Furthermore, by Theorem 2.0,

$$
\mathcal{L}\left(\mathfrak{X}_{A} \cdot x\right)=L^{* *}\left(\mathfrak{X}_{A} \cdot x\right)=\int_{H} \mathfrak{X}_{A} \cdot x d m=m(A) \cdot x,
$$

and $\left\|L_{A}\right\|_{(p, q)}=\tilde{m}_{(p, q)}(A)<\infty$ for appropriate pairings $(p, q), p \in\{p\}_{E}, q$ $\in\{p\}_{F}$. Also, if $z \in F^{*}$, then

$$
\begin{aligned}
\langle m(A) \cdot x, z\rangle & =\left\langle L^{* *}\left(\mathfrak{X}_{A} \cdot x\right), z\right\rangle \\
& =\mathfrak{X}_{A} \cdot x\left(L^{*}(z)\right)=\mu_{\left(x, L^{*}(z)\right)}(A),
\end{aligned}
$$

where $\mu_{\left(x, L L^{*}(z)\right)}$ is a finite regular Borel measure. The uniqueness statement follows from the corresponding uniqueness statement in the RRT.

We write $L \leftrightarrow m$ to denote the correspondence established in this theorem.

Next we show that the representation portion of Theorem 2.2 of [14] follows as an immediate corollary to Dinculeanu's representation theorem. To this end, let $H$ be an arbitrary point set, let $E$ and $F$ be normed linear spaces (NLS), and let $B$ be a linear space of bounded $E$-valued functions.

Theorem 2.3 [14, Theorem 2.2]. Suppose that $B$ is equipped with a norm $\|\cdot\|^{\prime}$ which is weaker than the uniform norm, and suppose there is an algebra $D$ of subsets of $H$ so that $\|\cdot\|^{\prime}$ can be extended to $\delta=S_{E}(D)$ in such a way that there is a subspace $\mathcal{S}^{\prime}$ of $\Phi$ satisfying

(i) $B \subset U\left(\mathcal{S}^{\prime}\right)$, the uniform closure of $\mathcal{S}^{\prime}$, and

(ii) there is a linear mapping $p: \delta \rightarrow B^{* *}$ which is continuous relative to $\|\cdot\|^{\prime} ;$ and, when we extend $\mathscr{P}$ to $U_{E}(\mathcal{D}), \mathcal{P}$ maps $\eta(f)$ into $f$ for each $f \in B$, where $\eta$ is the canonical embedding.

Then, if $T:\left(B\|\cdot\|^{\prime}\right) \rightarrow F$ is a continuous linear operator, there is a finitely additive set function $m: D \rightarrow B\left(E, F^{* *}\right)$ with finite semivariation $\tilde{m}$ so that $T(f)=\int_{H} f d m$, $f \in B$. Furthermore $\tilde{m}(A)=\left\|\left(T^{* *} \circ \mathscr{P}\right){ }_{A}\right\|, A \in \mathscr{D}$.

(We know that we can extend $\mathscr{P}$ to $U_{E}(\mathscr{D})$ since $\mathscr{P}$ is continuous on $\delta$ with respect to the uniform norm; we continue to denote the extension by $\mathscr{P}$.)

Proof. Since $9: U_{E}(\mathfrak{D}) \rightarrow B^{* *}$ is continuous relative to the uniform norm, then $T^{* *} \circ \mathscr{P}: U_{E}(\mathfrak{D}) \rightarrow F^{* *}$ is an operator. By Theorem 2.0 there is a unique finitely additive $m: \mathscr{D} \rightarrow B\left(E, F^{* *}\right)$ with finite semivariation so that

$$
T^{* *} \circ \mathscr{P}(g)=\int_{H} g d m, \quad g \in U_{E}(\mathfrak{D}),
$$

and

$$
\tilde{m}(A)=\left\|\left(T^{* *} \circ \mathscr{P}\right)_{A}\right\|, \quad A \in \mathscr{D} .
$$


But then if $f \in B$,

$$
T^{* *} \circ \mathscr{P}(f)=T^{* *}(f)=\int f d m,
$$

and we have the desired conclusion.

Definition $2.7[20, \S 1.5]$. Let $L: \mathbf{B}_{c} \rightarrow F$ be an operator. The point $t \in H$ will be in the support of $L$, denoted by $\operatorname{supp}(L)$, if and only if for each neighborhood $N(t)$ of $t$ there is an $f \in \mathbf{B}_{c}$ so that $\operatorname{supp}(f) \subset N(t)$ and $L(f) \neq 0$. If $L$ has $\mathbf{B}$ for its domain, the notion of support is defined similarly.

Theorem 2.4. Let $L: \mathbf{B}_{c} \rightarrow F$ be an operator so that $\operatorname{supp}(L)$ is compact. Then $L$ has a unique extension $\mathcal{L} \in B(C, F)$ so that $\mathcal{L}(f)=0$ for each $f \in C$ which vanishes in a neighborhood of $\operatorname{supp}(L)$.

The proof of this theorem very closely parallels the proof of the analogous statement in the scalar case and will be omitted.

Theorem 2.5. Let $F$ be a B-space. A linear mapping $\mathcal{L}: C \rightarrow F$ is continuous if and only if $\mathcal{L}$ is the unique extension of Theorem 2.4 of an operator $L \in B\left(\mathbf{B}_{c}, F\right)$ so that $L$ has compact support.

Proof. Suppose that $\mathcal{L} \in B(C, F)$. Then, by the continuity of $\mathcal{L}$, there is a compact set $K \subset H$, a positive number $M$, and a continuous seminorm $p$ on $E$ so that

$$
\|\mathcal{L}(x)\| \leq M p_{K}(x), \quad x \in C,
$$

where $p_{K}(x)=\sup \{p(x(t)): t \in K\}$. Now if $L=\left.L\right|_{B_{c}}$, then certainly $\operatorname{supp}(L)$ $C K$ and $L$ is an extension of an operator with compact support. If $x \in C$ vanishes in a neighborhood of $\operatorname{supp}(L)$, let $\varphi \in C_{c}(H)$ so that $0 \leq \varphi \leq 1$ and $\varphi(K)=1$. Then $x=\varphi \cdot x+(1-\varphi) \cdot x$, and

$$
\begin{aligned}
\hat{L}(x) & =\hat{L}(\varphi \cdot x)+\hat{L}((1-\varphi) \cdot x) \\
& =L(\varphi \cdot x)+\hat{L}((1-\varphi) \cdot x)
\end{aligned}
$$

But $f((1-\varphi) \cdot x)=0$ by (i), and $\varphi \cdot x \in \mathbf{B}_{c}$ so that $\varphi \cdot x$ vanishes on a neighborhood containing supp $(L)$. By appealing to a partition of unity argument, it follows that $L(\varphi \cdot x)=0$.

The converse of the theorem follows immediately from Theorem 2.4, and the proof is completed.

We remark that this characterization fails in case we allow $F$ to be an lcs.

Example 2.1. Let $H$ be a locally compact, noncompact, Hausdorff space, let $E=$ normed space, let $\mathbf{C}=C(H, E)$ with the compact-open topology, and let $F=\mathbf{C}$. If $I$ denotes the identity mapping from $\mathbf{C}$ to $\mathbf{C}$, then $\left.I\right|_{\mathbf{B}_{c}}$ is the identity mapping and obviously does not have compact support.

The following lemma enables us to see the measure theoretic consequences of Theorems 2.4 and 2.5 more clearly. 
Lemma 2.2 [23]. Suppose that $H, \Sigma, E$, and $F$ are as in the introduction. If $m: \Sigma \rightarrow B(E, F)$ is a weakly regular Borel measure, then there is a smallest closed set $D \subset H$ so that if $U$ is an open subset of $H$, then $\tilde{m}_{(p, q)}(U)=0$ for each $p \in\{p\}_{E}, q \in\{p\}_{F}$, if and only if $U \cap D=\varnothing$. Hence $m$ vanishes on $\Sigma$ $\cap(H-D)$.

This set $D$, called the support of $m$, is denoted by $\operatorname{supp}(m)$.

The proof of the following theorem involves primarily the technical details carried out in Lemma 2.1 and Theorem 2.1. Consequently, we omit the proof.

Theorem 2.6. Let $L: B \rightarrow F$ be an operator, with $L \leftrightarrow m$. Then $\operatorname{supp}(L)$ $=\operatorname{supp}(m)$.

We can now restate Theorem 2.5 as follows.

Corollary 2.6.1. If $F$ is a $B$-space, then a linear mapping $L: C \rightarrow F$ is an operator if and only if there is a unique weakly regular measure $m: \Sigma \rightarrow B\left(E, F^{* *}\right)$ so that $\operatorname{supp}(m)$ is compact and $L(f)=\int_{H} f d m, f \in \mathbf{C}$.

The following example is interesting in view of Theorem 2.2 of this paper.

Example 2.2. Let $H$ be the natural numbers equipped with the discrete topology, and let $B(H)$ be all the bounded, complex-valued functions on $H$ topologized by the uniform norm. Then $\Sigma$ is the power class of $H$. If $A \in \Sigma$, define $m(A)$ to be $\mathfrak{X}_{A} \in B(H) \cong B(\mathbb{C}, B(H))$. Then $m: \Sigma \rightarrow B(C, B(H))$ is finitely additive and has finite semivariation. If $f \in C(H)$, define $L(f)$ to be $\int_{H} f d m$.

Now let $\beta H$ denote the Stone-Čech compactification of $\boldsymbol{H}$, and choose $t \in \beta H-H$. For $f \in B(H)$, define $t(f)$ to be $\hat{f}(t)$, where $\hat{f}$ denotes the unique extension of $f$ to all of $\beta H$. Certainly $t \in B(H)^{*}$. We note next that $\hat{\mathfrak{X}}_{\left\{a_{i}\right\}}=\mathfrak{X}_{\left\{a_{i}\right\}}$ for a singleton $a_{i} \in H$. Therefore

$$
\sum_{i=1}^{\infty} m_{t}\left\{a_{i}\right\}=\sum_{i=1}^{\infty} t\left(\mathfrak{X}_{\left\{a_{i}\right\}}\right)=0 .
$$

But $m_{t}(H)=t\left(\mathfrak{X}_{H}\right)=1$, and $m_{t}$ is not countably additive. Hence the representing measure of Theorem 2.2 differs considerably from the measure $m$ used to define the operator $L$.

In the remainder of the paper, $E$ and $F$ will denote $B$-spaces.

We now turn to more general domain spaces $H$. As an application of Urysohn's extension theorem in [18] we obtain the following.

Lemma 2.3. If $H$ is a Hausdorff space, then $\left.C(H, E)\right|_{K}=C(K, E)$ for each compact $K \subset H$ if and only if the continuous functions on $H$ separate the points of $H$.

Such a Hausdorff space $H$ will be called an $S$-space.

Theorem 2.7. If $H$ is an S-space and $L: C=C(H, E) \rightarrow F$ is a linear transformation continuous with respect to the compact-open topology, then there is a 
unique weakly regular, compactly supported set function $m: \Sigma \rightarrow B\left(E, F^{* *}\right)$ so that $L(f)=\int_{H} f d m, f \in \mathbf{C}$.

Proof. Since $L$ is continuous, there is a constant $M>0$ and a compact set $K \subset H$ so that $\|L(f)\| \leq M\|f\|_{K}$, where $\|f\|_{K}=\sup \{\|f(t)\|: t \in K\}$. Therefore, by Theorem 2.5, $L$ induces an operator $T: C(K, E) \rightarrow F$. By Theorem 2.2, we may write $T(f)=\int_{K} f d m^{\prime}$, where $m^{\prime}: \Sigma(K) \rightarrow B\left(E, F^{* *}\right)$ is weakly regular. Since $\Sigma(H) \cap K=\Sigma(K)$, we may define $m: \Sigma(H) \rightarrow B\left(E, F^{* *}\right)$ by $m(A)$ $=m^{\prime}(A \cap K)$. Then clearly $m$ is weakly regular and $L(f)=\int_{H} f d m, f \in C$. The uniqueness follows from the uniqueness statement in Theorem 2.2.

In the future, we shall identify $m$ and $m^{\prime}$.

As an application of the following theorem, we can say more than simply that $m$ is weakly regular. In fact, it follows that if $m_{z}: \Sigma \rightarrow E^{*}$ is defined by $m_{z}(A) \cdot x=\langle m(A) \cdot x, z\rangle, x \in E$, then $\left|m_{z}\right| \in \operatorname{rca}(\Sigma, \mathbb{C})$.

Definition 2.8. If $H$ is a locally compact Hausdorff space, then an operator $L: B \rightarrow F$ is said to be dominated provided there is a positive linear functional $P \in C_{0}(H)^{*}$ so that $\|L(f)\| \leq P(\|f\|), f \in \mathbf{B}$.

The following theorem is due to N. Dinculeanu.

Theorem 2.8. If $L: B \rightarrow F$ is an operator with $L \leftrightarrow m$, then $L$ is dominated if and only if $|m| \in \operatorname{rca}(\Sigma, \mathbb{C})$.

Proof. If $m$ has finite total variation, which we denote by $|m|$, then certainly $\left\|\int f d m\right\| \leq \int\|f\| d|m|$. But $\int_{H}(\cdot) d|m|$ is a positive operator, and therefore $L$ is dominated.

Conversely, suppose that $L$ is dominated by the positive operator $P=\int(\cdot) d \mu$ ( $\mu$ is the positive regular Borel measure given by the RRT). Then, using the weak regularity of $m$ and repeating the construction in Lemma 2.1, it follows that $|m|(A) \leq \mu(A), A \in \Sigma$. But, from this inequality, it follows that $|m| \in \operatorname{rca}(\Sigma, \mathbf{C})$, and $m$ is countably additive.

We remark that if the set function given by Theorem 2.2 has finite total variation, then it must be countably additive.

Thus, if $L \leftrightarrow m$ as in Theorem 2.2 or Theorem 2.7, then $(z \circ L)(f)=z(L(f))$ $=\int_{H} f d m_{z},\|z \circ L\|=\tilde{m}_{z}(H)=\left|m_{z}\right|(H)$, and it follows that $\left|m_{z}\right| \in \operatorname{rca}(\Sigma, \mathbf{C})$ for each $z \in F^{*}$. We should also point out that $L^{*}(z)=m_{z}$. Therefore we have the following corollary.

Corollary 2.8.1. If $m: \Sigma \rightarrow B\left(E, F^{* *}\right)$ is finitely additive with finite semivariation and $m_{(x, z)}$ is a regular Borel measure for each $(x, z) \in E \times F^{*}$, then $\left|m_{z}\right|$ is a nonnegative regular Borel measure.

This prompts us to make the following definition.

Definition 2.9. By a representing measure $m: \Sigma \rightarrow B\left(E, F^{* *}\right)$ we shall mean a finitely additive set function with finite semivariation so that $\left|m_{z}\right|$ is a regular Borel measure for each $z \in F^{*}$. 
Remark. It is clear that an $S$-space $H$ is the most general domain space which will yield unique compactly supported representing measures. In the $C_{0}(H, E)$ setting, the support of a representing measure need only be closed and not necessarily compact.

3. Weak compactness of vector measures. In this section we establish criteria for weak compactness in the $B$-space of vector measures. This will enable us to determine the compactness or weak compactness of operators on function spaces by examining the weak compactness of the set of vector measures formed by the image of the unit sphere under the adjoint operator. The various results concerning weak compactness of vector measures are due to Brooks and are essentially taken from [6], where the proofs are briefly outlined (for the reflexive case). We are grateful to $\mathrm{C}$. Swartz for providing us with Lemma 3.3 which enabled us to eliminate a weak sequential completeness condition that was imposed earlier.

The following definition is fundamental in characterizing representing measures of weakly compact operators.

Definition 3.1. Let $\mathfrak{D}$ be a ring of subsets of a set $S$. A finitely additive set function $m: \mathfrak{D} \rightarrow B\left(E, F^{* *}\right)$ is strongly bounded (s-bounded) if $\tilde{m}\left(A_{i}\right) \rightarrow 0$, whenever $\left(A_{i}\right)$ is a disjoint sequence of sets from $\mathfrak{D}$. An operator $L$ is said to be $s$ bounded provided that its representing measure is $s$-bounded.

A similar concept was introduced by Lewis [22] under the name variational semiregularity (vsr), where the requirement took the form $\tilde{m}\left(A_{i}\right) \rightarrow 0$, where $A_{i}$ are Borel sets satisfying $A_{i} \searrow \varnothing$. We remark that countable additivity, even on a $\sigma$-algebra, need not imply $s$-boundedness (Lewis [22]), as Example 5.1 infra will show. Rickart [28] also introduced a continuity condition in a different setting.

Let $X$ be a $S$-space. By fa $(\mathfrak{D}, X)$ we denote the Banach space of all finitely

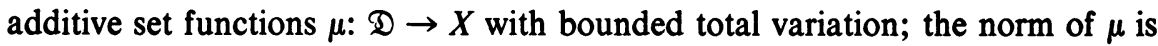
given by $|\mu|(S)$. The subspace consisting of countably additive measures is denoted by ca $(\mathfrak{D}, X)$.

A set is said to be conditionally compact with respect to a topology $\tau$ if its $\tau$ closure is $\tau$-compact. In the sequel, we shall use the following equality [11]:

$$
\tilde{m}(A)=\sup \left\{\left|m_{z}\right|(A): z \in F_{1}^{*}\right\}, \quad A \in \mathfrak{D} .
$$

Lemma 3.1. Suppose that $D$ is an $S$-space and $m: \Sigma(D) \rightarrow B(E, F)$ is a representing measure. Then the following are equivalent:

(i) $m$ is $s$-bounded;

(ii) if $A_{n} \searrow \varnothing$, then $\tilde{m}\left(A_{n}\right) \rightarrow 0$;

(iii) $\left\{\left|m_{z}\right|: z \in F_{1}^{*}\right\}$ is conditionally weakly compact in $\mathrm{ca}\left(\Sigma, F^{*}\right)$;

(iv) $\sum m\left(A_{i}\right) x_{i}$ converges in $F$ for each disjoint sequence $\left(A_{i}\right)$ and $\left(x_{i}\right) \subset E_{1}$.

Proof. (ii) $\Rightarrow$ (i). Let $\left(A_{i}\right)$ be a disjoint sequence. Then $B_{k} \searrow \varnothing$, where $B_{k}=\cup_{n \geq k} A_{n}$. Hence $\tilde{m}\left(A_{k}\right) \leq \tilde{m}\left(B_{k}\right) \rightarrow 0$ and $m$ is $s$-bounded. 
(ii) $\Rightarrow$ (iii). In view of equality (III.1), $m$ satisfies (ii) if and only if $\left\{\left|m_{z}\right|: z \in F_{1}^{*}\right\}$ is uniformly countably additive. The result follows by Theorem IV.9.1 in [13].

(i) $\Rightarrow$ (iv). Let $\left(A_{i}\right)$ be a disjoint sequence, $\left(x_{i}\right) \subset E_{1}$ and suppose that $\sum m\left(A_{i}\right) x_{i}$ does not converge. Then there is a subsequence $\left(i_{k}\right)$ and an $\varepsilon>0$ such that

$$
\left\|\sum_{n=i_{k}+1}^{i_{k+1}} m\left(A_{n}\right) x_{n}\right\|>\varepsilon, \quad k=1,2, \ldots
$$

Let $D_{k}=\bigcup_{n=l_{k}+1}^{i_{k+1}} A_{n}$. Then $\left(D_{k}\right)$ is a disjoint sequence and $\tilde{m}\left(D_{k}\right)>\varepsilon$ for each $k$. Hence $m$ is not $s$-bounded.

(iv) $\Rightarrow$ (i). If $m$ is not $s$-bounded, there exists an $\varepsilon>0$ and a disjoint sequence $\left(A_{i}\right)$ such that $\tilde{m}\left(A_{i}\right)>\varepsilon$ for each $i$. From the definition of $\tilde{m}$, there exist partitions $\left(B_{k}^{i}\right)_{k=1}^{n(i)}$ of $A_{i}$ and $\left(x_{k}^{i}\right)_{k=1}^{n(i)} \subset E$ so that $\left\|\sum_{k} m\left(B_{k}^{i}\right) x_{k}^{i}\right\|>\varepsilon$ for each $i$. Consequently (iv) does not hold.

(i) $\Rightarrow$ (ii). If (ii) does not hold, there exists an $\varepsilon>0$ and $A_{i} \searrow \varnothing$ such that $\tilde{m}\left(A_{i}\right)>\varepsilon$. There is a $z_{1} \in F_{1}^{*}$ such that $\left|m_{z_{1}}\right|\left(A_{1}\right)>\varepsilon$. Choose $N_{2}>N_{1}=1$ so that $\left|m_{z_{1}}\right|\left(A_{N_{1}}-A_{N_{2}}\right)>\varepsilon$. Proceeding inductively, we obtain an increasing sequence $\left(N_{i}\right)$ so that $\tilde{m}\left(A_{N_{i}}-A_{N_{i+1}}\right)>\varepsilon, i=1,2, \ldots$ Thus $m$ is not $s$-bounded. This completes the proof of the lemma.

Definition 3.2. A $B$-space $X$ has the Radon-Nikodym property (property R-N) if every countably additive $X$-valued measure $m$ of bounded variation defined on a $\sigma$-algebra, which is absolutely continuous with respect to a scalar measure $\nu$, can be expressed as the indefinite integral of a Bochner integrable function $f$; in symbols, $d m / d \nu=f \in L^{1}(\nu, X)$.

Remark 3.1. The following spaces are known to have property R-N: reflexive spaces [27] and separable dual spaces [12].

The next result is a key lemma in obtaining criteria for weak compactness in the countably additive case. We denote weak convergence by $\rightarrow$ " and uniform convergence by $\rightarrow$.

Lemma 3.2. Let $Q$ be $a$ o-algebra and let $X$ be $a$ B-space such that $X$ and $X^{*}$ have property $R-N$. Suppose that $\left(\mu_{n}\right)$ is a sequence in $\mathrm{ca}(\mathbb{Q}, X)$ such that $\sup _{n}\left|\mu_{n}\right|(S)$ $=M<\infty$. Assume that $\left\{\left|\mu_{n}\right|: n=1,2, \ldots\right\}$ is uniformly countably additive and $\mu_{n}(A) \rightarrow^{w} \mu(A)$ in $X$ for each $A \in \mathbb{Q}$. Then $\mu: \mathbb{Q} \rightarrow X$ belongs to ca $(\mathbb{Q}, X)$ and $\mu_{n} \rightarrow^{w}$ $\mu$ in $\mathrm{ca}(\mathbb{Q}, X)$.

Proof. Let $x^{*} \in X^{*}$. Since $x^{*} \mu_{n}(A) \rightarrow x^{*} \mu(A)$ for each $A, x^{*} \mu$ is countably additive by the Nikodym theorem. It then follows from the Orlicz-Pettis theorem that $\mu$ is countably additive. Let $\left(A_{i}\right)_{i=1}^{m}$ be a partition of $S$. For suitable $x_{i}^{*} \in X_{1}^{*}$,

$$
\begin{aligned}
\sum_{i=1}^{m}\left\|\mu\left(A_{i}\right)\right\| & =\sum_{i=1}^{m}\left|x_{i}^{*} \mu\left(A_{i}\right)\right|=\lim _{k} \sum_{i=1}^{m}\left|x_{i}^{*} \mu_{k}\left(A_{i}\right)\right| \\
& \leq \lim _{k} \sum_{i=1}^{k}\left\|\mu_{k}\left(A_{i}\right)\right\| \leq M .
\end{aligned}
$$


Since $|\mu|(S) \leq M, \mu \in \operatorname{ca}(Q, X)$. Let $\mu_{0}=\mu$ and define $\nu(A)=\sum_{i=0}^{\infty}\left|\mu_{i}\right|(A) / 2^{i}$, $A \in Q$. Note that $\nu$ is finite, countably additive and $\mu \ll \nu$ for each $i$. Since $X$ has property R-N, for each $i$ there exists an $f_{i} \in L^{1}(\nu, X)$ such that $d \mu_{i} / d \nu=f_{i}$. To show that $\mu_{i} \rightarrow^{w} \mu_{0}$, it suffices to prove that $f_{i} \rightarrow^{w} f_{0}$ in $L^{1}(\nu, X)$. Since $X^{*}$ has property $\mathrm{R}-\mathrm{N}$, one can show that $L^{1}(\nu, X)^{*}=L^{\infty}\left(\nu, X^{*}\right)$. Consequently, we have to show that $\int_{s} \varphi f_{i} \rightarrow \int_{s} \varphi f_{0}$ for each $\varphi \in L^{\infty}\left(\nu, X^{*}\right)$. To establish this, choose a sequence $\left(\psi_{n}\right)$ of $Q$-simple $X^{*}$-valued functions satisfying $\psi_{n} \rightarrow \varphi$ a.e. $\nu$ and $\left\|\psi_{n}(t)\right\|<2\|\varphi(t)\|$ for each $n$. Since $\left|\mu_{i}\right| \ll \nu$ uniformly in $i$ (see [8, Theorem 1]), given an $\varepsilon>0$ there exists a $\delta>0$ such that $\left|\mu_{i}\right|(A)<\varepsilon, i=1,2, \ldots$, if $\nu(A)<\delta$. By Egoroff's theorem, there is an $A \in \mathbb{Q}$ such that $\nu(A)<\delta$, and $\Psi_{i} \rightarrow^{u} \varphi$ on $S-A$. By considering the inequality

$$
\begin{aligned}
\left|\int \varphi f_{i}-\int \varphi f_{0}\right| \leq & \left|\int \Psi_{n} f_{i}-\int \varphi f_{i}\right| \\
& +\left|\int \Psi_{n} f_{i}-\int \Psi_{n} f_{0}\right|+\left|\int \Psi_{n} f_{0}-\int \varphi f_{0}\right|
\end{aligned}
$$

one can show that $\left|\int \varphi f_{i}-\int \varphi f_{0}\right|<K \varepsilon$ for $i \geq I_{\varepsilon}$ and a suitable constant $K$. The lemma then follows.

Proposition 3.1. (i) Assume that $X$ and $X^{*}$ have property $R-N$. Let $\nVdash \subset \mathrm{ca}(\mathbb{Q}, X)$ satisfy the following conditions:

(a) $\pi$ is bounded;

(b) $\{|\mu|: \mu \in \mathcal{K}\}$ is uniformly countably additive;

(c) $\{\mu(A): \mu \in \Re\}$ is conditionally weakly compact in $X$ for each $A \in \mathbb{Q}$.

Then $\Re$ is conditionally weakly compact in $\mathrm{ca}(\mathbb{Q}, X)$.

(ii) Conversely, if $X$ is any $B$-space and $\Re \subset \mathrm{ca}(\mathbb{Q}, X)$ is conditionally weakly compact, then (a), (b), and (c) hold.

Proof. To prove (i), it suffices in view of the Eberlein-Šmulian theorem to prove

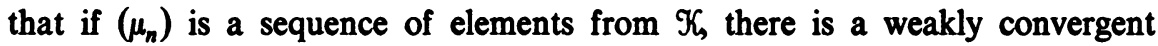
subsequence. Construct a countable subalgebra $\mathbb{Q}_{0}=\left\{A_{1}, A_{2}, \ldots\right\}$ of $\mathbb{Q}$ such that $\left|\mu_{n}\right|(S)=\left|\mu_{n}\right|_{\theta_{0}} \mid(S), n=1,2, \ldots$ Let $\mathbb{Q}^{\prime}$ be the $\sigma$-algebra generated by $\mathbb{Q}_{0}$. Thus the mapping $\pi: \operatorname{span}\left(\mu_{n}\right) \rightarrow \mathrm{ca}\left(\mathbb{Q}^{\prime}, X\right)$ is an isometry, where $\pi(\mu)=\left.\mu\right|_{\mathscr{Q}^{\prime}}$. Using (c) we can choose a subsequence $\left(\beta_{k}\right)$ of $\left(\mu_{k}\right)$ such that $\beta_{k}\left(A_{n}\right) \rightarrow_{k}^{w}, n=1,2, \ldots$. By Theorem 4 in [5], $x^{*} \beta_{k}(A) \rightarrow$ for every $A \in \mathbb{Q}^{\prime}$ and $x^{*} \in X^{*}$. Using (c) we conclude that $\beta_{k}(A) \rightarrow^{w}$ for every $A \in \mathbb{Q}^{\prime}$. If we define $\beta_{0}(A)$ to be this weak limit, then $\pi \beta_{k} \rightarrow{ }^{w} \beta_{0}$ in ca $\left(\mathbb{Q}^{\prime}, X\right)$ by Proposition 3.1. Hence $\beta_{k} \rightarrow^{w} \pi^{-1}\left(\beta_{0}\right)$ in ca $(Q, X)$.

To prove the converse, assume that $\mathcal{K}$ is conditionally weakly compact and $\{|\mu|: \mu \in \mathscr{K}\}$ is not uniformly countably additive. There exists an $\varepsilon>0,\left(\mu_{i}\right)$ $\subset \mathcal{K}$ and a disjoint sequence $\left(A_{i}\right)$ such that $\left|\mu_{i}\right|\left(A_{i}\right)>\varepsilon, i=1,2, \ldots$ Obtain partitions $\left\{B_{i j}\right\}_{j=1}^{n(i)}$ of $A_{i}$ and $x_{i j}^{*} \in X_{1}^{*}$ such that

$$
\sum_{j=1}^{n(i)}\left|x_{i j}^{*} \mu_{i}\left(B_{i j}\right)\right|>\varepsilon, \quad i=1,2, \ldots
$$


The map $\Upsilon: \mathcal{K} \rightarrow l_{1}$ defined by $\Upsilon(\mu)=\left\{x_{i j}^{*} \mu\left(B_{i j}\right)\right\}_{j=1 ; i=1}^{n(i) ; \infty} \in l_{1}$ is continuous with respect to weak topologies on $\mathscr{K}$ and $l_{1}$. Thus $\Upsilon(\mathcal{K})$ is a conditionally weakly compact subset of $l_{1}$; by the Schur theorem, $\Upsilon(\mathcal{K})$ is conditionally strongly compact. One can show that in view of (\#), this leads to a contradiction. This completes the proof of the proposition.

We now turn our attention to the finitely additive case. A family $\Re$ of finitely additive set functions $\mu: \mathfrak{D} \rightarrow X$ is uniformly strongly additive if $\mu\left(A_{i}\right) \rightarrow 0$ uniformly with respect to $\mu \in \mathscr{K}$ whenever $\left(A_{i}\right)$ is a disjoint sequence. When $\mathscr{K}$ consists of countably additive measures and $\mathfrak{D}$ is a $\sigma$-algebra, this concept coincides with uniform countable additivity.

First we need the following lemma.

Lemma 3.3. Let I be a nonempty set. Suppose that there exists a sequence of functions $f_{n}: I \rightarrow X$ such that $f_{n}(I)$ is a conditionally weakly compact set in $X$ for each $n$. Assume that $f: I \rightarrow X$ and $\lim _{n}\left\|f_{n}(\alpha)-f(\alpha)\right\|=0$ uniformly for $\alpha \in I$. Then $f(I)$ is conditionally weakly compact.

Proof. Let $B(I)$ denote the $B$-space of all bounded functions with norm defined by the sup norm. Let $f \equiv f_{0}$ and for each $n \geq 0$ define $T_{n}: X^{*} \rightarrow B(I)$ by $T_{n}\left(x^{*}\right)=x^{*} f_{n}(\cdot)$. By Theorem 2 in [33], each $T_{n}$ is a weakly compact operator. Since $T_{n} \rightarrow T$ in the uniform operator topology, $T$ is weakly compact. Again by the above-mentioned theorem, $f(I)$ is conditionally weakly compact in $X$.

We can now state the main theorem on weak compactness in $\mathrm{fa}(\mathfrak{D}, X)$.

Theorem 3.1. (i) Assume that $X$ and $X^{*}$ have property $R-N$. Let $\mathscr{K} \subset \mathrm{fa}(\mathfrak{D}, X)$ satisfy the following conditions:

(a) $\mathcal{K}$ is bounded;

(b) $\{|\mu|: \mu \in \mathscr{K}\}$ is uniformly strongly additive;

(c) $\{\mu(A): \mu \in \mathscr{K}\}$ is conditionally weakly compact in $X$ for each $A \in \mathfrak{D}$.

Then $\nVdash$ is conditionally weakly compact in $\mathrm{fa}(\mathfrak{D}, X)$.

(ii) Conversely, if $X$ is any $B$-space and $\nVdash \subset \mathrm{fa}(\mathfrak{D}, X)$ is conditionally weakly compact, then (a), (b) and (c) hold.

Remark 3.2. In view of Remark 3.1, if $X$ is reflexive or $X=Y^{*}$ and $X^{*}$ is separable, then $X$ and $X^{*}$ have property R-N and thus satisfy the hypotheses of (i).

Proof. Assume $\mathscr{D}=\Re$ is an algebra of sets-that is, $S \in \Re_{\text {. Let }} \Re_{1}$ be the Stone algebra [13, p. 312] of all open-closed subsets of the compact Hausdorff space $S_{1}$. The $\sigma$-algebra generated by $\Re_{1}$ is denoted by $\Re_{2}$. We shall use the fact that $\mathrm{fa}(\Re, X)$ and $\mathrm{ca}\left(\Re_{2}, X\right)$ are isometrically isomorphic (see [11]). Let $\mathcal{K}^{\prime}$ denote the usual image of $\Re$ in ca $\left(\Re_{1}, X\right)$; $\Re^{\prime \prime}$ denotes the extensions of $\mathcal{K}^{\prime}$ to $R_{2}$. It suffices to show that $\mathcal{K}^{\prime \prime}$ satisfies (a), (b) and (c) of Proposition 3.1 if $\mathcal{K}$ satisfies the hypotheses of (i). Clearly (a) is satisfied. Next, (b) follows from Theorem 3 in [5], which implies that since $\left\{\left|\mu^{\prime}\right|: \mu^{\prime} \in \mathscr{K}\right\}$ is uniformly strongly additive on $\Re_{1}$, 
$\left\{\left|\mu^{\prime \prime}\right|: \mu^{\prime \prime} \in \mathcal{K}^{\prime \prime}\right\}$ is uniformly countably additive on $\mathscr{R}_{2}$. Note that we use the fact that the $\left|\mu^{\prime \prime}\right|$ is the extension of $\left|\mu^{\prime}\right|$. To prove (c), let $A \in \Re_{2}$ and let $\left(\sigma_{n}\right) \equiv\left(\mu_{n}^{\prime \prime}\right) \subset \mathcal{K}^{\prime \prime}$. Set $\lambda=\sum\left|\sigma_{n}\right| / 2^{n}$. Then $\lambda$ is a bounded measure such that $\left|\sigma_{n}\right| \ll \lambda$ for each $n$. By Theorem 1 in [8], the $\sigma_{n}$ are uniformly absolutely continuous with respect to $\lambda$. Choose a sequence of sets $B_{n} \in \mathscr{R}_{1}$ such that $\lambda\left(B_{n} \Delta A\right) \rightarrow 0$. Hence $\sigma_{i}\left(B_{n}\right) \rightarrow_{i} \sigma_{i}(A)$ uniformly in $i$. Let $I$ be the set of positive integers. Define $f, f_{n}: I \rightarrow \mathfrak{X}$ by $f_{n}(i)=\sigma_{i}\left(B_{n}\right), f(i)=\sigma_{i}(A)$. Since each $f_{n}(I)$ is conditionally weakly compact and $f_{n} \rightarrow f$ uniformly on $I$, we conclude by Lemma 3.3 that $f(I)=\left\{\sigma_{i}(A): i=1,2, \ldots\right\}$ is conditionally weakly compact. This establishes (c).

In the general case when $\mathscr{D}$ is a ring, we consider $\Re$ the algebra generated by $\mathscr{D}$. Then $\mathcal{K}$ is extended to $\mathcal{K}^{\prime}$ on $\Re$ in such a way that (a), (b) and (c) hold for $\mathcal{K}^{\prime}$ on $\Re$ (see 3.V in [6]). The problem is then reduced to the above case. This completes the proof of the theorem.

4. Weakly compact and compact operators. In this section we establish criteria for operators to be (weakly) compact on certain function spaces.

Definition 4.1. Let $T: C(H, E) \rightarrow F$ be an operator. We say that $T$ is (weakly) compact if there exists a compact set $K$ such that $T\left(\left\{f:\|f\|_{K} \leq 1\right\}\right)$ is conditionally (weakly) compact in $F$.

Theorem 4.1. Let $L$ be an operator defined on either $C(H, E)$ or $C_{0}(H, E)$ into $F$, with $L \leftrightarrow m$. If $L$ is weakly compact then $m$ is s-bounded, and $m(A): E \rightarrow F$ is weakly compact for each $A$.

Conversely, if $E^{*}$ and $E^{* *}$ have property $R-N$ (e.g. if $E$ is reflexive or $E^{* *}$ is separable), $m$ is s-bounded and $m(A)$ is a weakly compact operator in $B(E, F)$ for each $A$, then $L$ is weakly compact.

Proof. We shall only consider the case where $L$ is defined on $C(H, E)$. Suppose $L$ is weakly compact. One can find a compact set $K \subset H$ and a constant $M>0$ such that $\|L(f)\| \leq M\|f\|_{K}, f \in C(H, E)$ and $L\left(\left\{f:\|f\|_{K} \leq 1, f \in C(H, E)\right\}\right)$ is conditionally weakly compact in $F$. Thus we may consider $L$ as an operator on $C(K, E)$ into $F$. By Gantmacher's theorem,

$$
L^{*}: F^{*} \rightarrow C^{*}(K, E) \subset \mathrm{ca}\left(\Sigma(H), E^{*}\right)
$$

(see Corollary 2.9.1) is weakly compact. Consequently, $\left\{m_{z}: z \in F_{1}^{*}\right\}=\left\{L^{*}(z)\right.$ : $\left.z \in F_{1}^{*}\right\}$ is conditionally weakly compact in $\mathrm{ca}\left(\Sigma, E^{*}\right)$. By Proposition 3.1, $\left\{\left|m_{z}\right|: z \in F_{1}^{*}\right\}$ is uniformly countably additive, hence conditionally weakly compact in $\mathrm{ca}(\Sigma, C)$. By Lemma 3.1, $m$ is $s$-bounded.

Conversely, suppose the conditions of the second part of the theorem are fulfilled. As before we regard $L$ as an operator on $C(K, E)$ for some compact set $K$. Consider the set $\mathscr{K}=\left\{m_{z}: z \in F_{1}^{*}\right\}=L^{*}\left(F_{1}^{*}\right)$. It suffices to show that $\mathcal{K}$ is conditionally weakly compact in $\mathrm{ca}\left(\Sigma(K), E^{*}\right)$. But $\mathscr{K}$ is bounded since $\tilde{m}(K)$ $<\infty$, and $\left\{\left|m_{z}\right|: z \in f_{1}^{*}\right\}$ is uniformly countably additive since $m$ is $s$-bounded. Let $A \in \Sigma(K)$. Since $m(A): E \rightarrow F$ (see Corollary 4.4.1 infra) is weakly compact, $\left\{m(A)^{*} z: z \in F_{1}^{*}\right\}=\left\{m_{z}(A): z \in F_{1}^{*}\right\}$ is conditionally weakly compact in $E^{*}$. The conditional weak compactness of $\Re$ now follows from Proposition 3.1. 
Remark 4.1. It follows from the above theorem that if $L$ is weakly compact, then $m$ is countably additive. If $L$ is a dominated operator, then $|m|$ is finite and countably additive, hence $s$-bounded. Thus in this case $L$ is weakly compact if $E^{*}$ and $E^{* *}$ have property R-N and $m(A)$ is a weakly compact operator for each $A$. The above theorem strengthens Theorem VI.7.3 of [13] as follows: $L: C_{0}(H)$ $\rightarrow F$ is weakly compact if and only if $m$ is countably additive. The hypothesis that $m$ maps into $F$ is unnecessary. We mention that if the hypotheses on $E^{*}$ and $E^{* *}$ are omitted in the above theorem, then the result is false. In fact if $E$ is not reflexive, one can exhibit nonweakly compact operators which correspond to $s$ bounded measures. Special cases of the above result have been obtained by Batt and Berg [3] and C. Swartz (private communication).

Next we consider operators on the space of totally measurable functions $U_{E}(\mathfrak{D})$ (Definition 2.1) into $F$. First we state a lemma whose proof will be left to the reader.

Lemma 4.1. Let $L: U_{E}(\mathfrak{D}) \rightarrow F$ be an operator. Then there exists a unique finitely additive set function $m: \mathfrak{D} \rightarrow B(E, F)$ such that $\tilde{m}(S)=\|L\|<\infty$ and $L(f)$ $=\int f d m, f \in U_{E}(\mathfrak{D})$.

If $L$ and $m$ are related as above, we write $L \leftrightarrow m$. The following theorem characterizes weakly compact operators on $U_{E}(\mathfrak{D})$.

Theorem 4.2. Let $L: U_{E}(\mathfrak{D}) \rightarrow F$ be an operator, with $L \leftrightarrow m$. If $L$ is weakly compact, then $m$ is $s$-bounded.

Conversely, if $E^{*}$ and $E^{* *}$ have property $R-N$ (e.g. if $E$ is reflexive or $E^{* *}$ is separable), $m$ is s-bounded and $m(A): E \rightarrow F$ is weakly compact for each $A \in \mathfrak{D}$, then $L$ is weakly compact.

Proof. We first note that $U_{E}^{*}(\mathfrak{D}) \subset \mathrm{fa}\left(\mathfrak{D}, E^{*}\right)$. Since $L^{*}\left(F_{1}^{*}\right)=\left\{m_{z}: z \in F_{1}^{*}\right\}$, the theorem follows in view of Theorem 3.1.

Using Theorem 4.2 we establish a result concerning the pointwise limit of weakly compact operators on the space of totally measurable functions.

Theorem 4.3. (i) If $c_{0} \oplus F$ and $L: U_{E}(\mathfrak{D}) \rightarrow F$ is an operator, then $L$ is $s-$ bounded. (ii) Let $E^{*}$ and $E^{* *}$ have property $R-N$, let $L: U_{E}(\mathfrak{D}) \rightarrow F$ be an operator, let $L_{n}$ be a sequence of weakly compact operators, and suppose that $c_{0} \nsubseteq F$. If $L_{n}\left(\mathfrak{X}_{A} \cdot x\right) \rightarrow L\left(\mathfrak{X}_{A} \cdot x\right)$ for each $A$ and uniformly for $x \in E_{1}$, then $L$ is weakly compact.

Proof. (i) Suppose that $L$ is not $s$-bounded. Then there is a disjoint sequence $\left(A_{i}\right)$, a subsequence $\left(n_{k}\right)$ of positive integers, a sequence $\left(x_{i}\right) \subset E_{1}$, and an $\varepsilon>0$ so that

$$
\left\|\sum_{i=n_{k}+1}^{n_{k+1}} m\left(A_{i}\right) \cdot x_{i}\right\|>\varepsilon
$$


However, if we select $z \in F_{1}^{*}$, then $\sum_{i=1}^{\infty}\left|\left\langle m\left(A_{i}\right) x_{i}, z\right\rangle\right| \leq \sum_{i=1}^{\infty}\left|m_{z}\right|\left(A_{i}\right)$ $\leq \sup \left\{\left|m_{z}\right|(A): A \in \mathfrak{D}\right\} \leq \sup \{\tilde{m}(A): A \in \mathfrak{D}\}<\infty$, and (\#) is a weakly unconditionally convergent series which is not unconditionally convergent. Thus by Bessaga and Pelczyński [4, p. 160], $F$ contains $c_{0}$, and (i) is proved.

(ii) Let $m_{n} \leftrightarrow L_{n}$ and $m \leftrightarrow L$. Since $m_{n}(A) x=L_{n}\left(\mathfrak{X}_{A} \cdot x\right) \rightarrow m(A) x=$ $L\left(\mathfrak{X}_{A} \cdot x\right)$ for each $A$ and uniformly for $x \in E_{1}$, then $m(A)$ is a weakly compact operator for each $A$. And, since $m$ is $s$-bounded by (i), the result follows from the preceding theorem.

Next we turn to the problem posed by Dinculeanu $[11, \mathrm{p} .416]$ of characterizing those operators $L: B \rightarrow F$ whose representing measures actually map into $B(E, F)$. This question has also been studied in [3] and [16]. We give a characterization analogous to the theory developed by Bartle, Dunford and Schwartz [2].

If $L: C_{0}(H, E) \rightarrow F$ is an operator and $x \in E$, let $L_{x}: C_{0}(H) \rightarrow F$ be the usual induced operator. Note that $L^{* *}\left(X_{A} \cdot x\right)=L_{x}^{* *}\left(X_{A}\right), A \in \Sigma(H)$. For $x \in E$ and $y \in F^{* * *}, m_{x}$ and $m_{y}$ are defined in the obvious manner.

Theorem 4.4. Let $L: B \rightarrow F$ be an operator with $L \leftrightarrow m$. Then the following are equivalent:

(i) $m: \Sigma \rightarrow B(E, F)$;

(ii) $m_{x}$ is countably additive for each $x \in E$;

(iii) $L_{x}: C_{0}(H) \rightarrow F$ is weakly compact for each $x \in E$;

(iv) $m_{y}$ is countably additive for each $y \in F^{* * *}$.

Proof. (iii) $\Rightarrow$ (i). Since $L_{x} \leftrightarrow m_{x}$ and $L_{x}^{* *}: U_{\mathrm{c}}(\Sigma) \rightarrow F$, we have $m_{x}(A) \in F$ for each $x \in E$. Therefore $m: \Sigma \rightarrow B(E, F)$.

(i) $\Rightarrow$ (ii). Since $m_{x}: \Sigma \rightarrow F$ and $m_{x}$ is weakly countably additive, by the OrliczPettis theorem $m_{x}$ is countably additive.

(ii) $\Rightarrow$ (iii). If $m_{x}$ is countably additive for each $x$, it follows that $m_{x}$ is $s$ bounded, and thus $L_{x}$ is weakly compact by Theorem 4.1.

(iv) $\Rightarrow$ (ii). Since $m_{y}$ is countably additive for each $y \in F^{* * *}$, it follows that $m_{x}$ is weakly countably additive, and by the Orlicz-Pettis theorem $m_{x}$ is countably additive.

(i) $\Rightarrow$ (iv). Since $m: \Sigma \rightarrow B(E, F)$, it follows that if $y \in F^{* * *}$ and $z=\left.y\right|_{F}$, then $m_{y}=m_{z}$. Therefore $m_{y}$ is countably additive since $\left|m_{z}\right| \in \operatorname{rca}(\Sigma, \mathbb{C})$.

The next corollary follows from the above theorem.

Corollary 4.4.1. Let $m$ be a representing measure. Then $m$ takes its values in $B(E, F)$ in any of the following cases: (a) $m$ is countably additive; (b) $m$ is s-bounded; (c) $m$ corresponds to a weakly compact operator.

We remark here that the weak extension $\hat{L}: U_{E}(\Sigma) \rightarrow F$ of $L$ which is given in Theorem 1(b) of Batt and Berg [3] is simply $L^{* *}$. Therefore if $L \leftrightarrow m$, then it is immediate that $L$ is (weakly) compact if and only if $m: \Sigma \rightarrow B(E, F)$ and 
$A=\left\{\sum_{\pi(H)} m\left(A_{i}\right) x_{i}: x_{i} \in E_{1}\right\}$ is (weakly) conditionally compact. This is the principal content of Theorem 6 of [3].

We mention that if $L \leftrightarrow m$ and $m$ is $s$-bounded, then $m$ is variationally regular. That is, if $\varepsilon>0$ and $A \in \Sigma$, then there is a compact set $K \subset A$ and an open set $U \supset A$ so that $\tilde{m}(U-K)<\varepsilon$. This follows since there is a positive $\lambda \in \operatorname{rca}(\Sigma, \mathbb{C})$ so that $\left|m_{z}\right|(A) \rightarrow 0$ as $\lambda(A) \rightarrow 0$ uniformly for $z \in F_{1}^{*}$.

We next turn our attention to compact operators on function spaces.

Definition 4.2. Suppose that $\mathfrak{D}$ is an arbitrary ring of subsets of $S$ and $m: \mathfrak{D} \rightarrow B(E, F)$ is finitely additive with finite semivariation. If $A \in \mathfrak{D}$ and $z \in F^{*}$, then let $p_{(m, A)}(z)=\left|m_{z}\right|(A)$. The topology induced on $F^{*}$ by the seminorms $\left\{p_{(m, A)}: A \in \mathfrak{D}\right\}$ will be denoted by $\delta(m)$. We note that if $S \in \mathfrak{D}$, then $\left(F^{*}, \delta(m)\right)$ is a pseudometric space whose topology is determimed by $p_{(m, S)}$.

It is known that if $H$ is compact and $L: C(H) \rightarrow F$, with $L \leftrightarrow m$, where $L$ is an operator, then $L$ is compact if and only if $m$ takes its values in a compact subset of $F$ [13]. However, if $L$ has $C(H, E)$ as its domain, then the range of $m$ need not be compact if $L$ is compact [3]. Conversely, Example 5.1 infra shows that if the range of $m$ is compact and for each $A, m(A)$ is compact (even nuclear), then $m$ may not be $s$-bounded; hence $L$ is not even weakly compact.

Theorem 4.5. Let $L: C(H, E) \rightarrow F$ be an operator, with $L \leftrightarrow m$. Then

(i) $L$ is compact if and only if $\left(F_{1}^{*}, \delta(m)\right)$ is a compact pseudometric space;

(ii) $L$ is compact with dense range if and only if $\delta(m)$ induces the $w^{*}$-topology on $F_{1}^{*}$.

Proof. Suppose $L: C(H, E) \rightarrow F$ is a compact operator. As we have seen before, we may consider $L: C(K, E) \rightarrow F$, where $K$ is a compact set. To show $\left(F_{1}^{*}, \delta(m)\right)$ is a compact space, let $\left(z_{\alpha}\right)$ be a net in $F_{1}^{*}$. By the Banach-Alaoglu theorem, there is a subnet $\left(z_{\alpha^{\prime}}\right)$ of $\left(z_{\alpha}\right)$ converging to $z \in F_{1}^{*}$ in the $w^{*}$-topology. By Theorem VI.5.6 in [13], $L^{*}\left(z_{\alpha^{\prime}}\right) \rightarrow L^{*}(z)$ in ca $\left(\Sigma, E^{*}\right)$. This means that $z_{\alpha^{\prime}} \rightarrow z$ in $\left(F_{1}^{*}, \delta(m)\right)$.

Conversely, suppose that $\left(F_{1}^{*}, \delta(m)\right)$ is a compact space. Let $\left(z_{n}\right)$ be a sequence in $F_{1}^{*}$. There exists a subsequence $\left(z_{n_{i}}\right)$ such that $\left|m_{\left(z_{m}-z\right)}\right|(K) \rightarrow 0$. This implies that $L^{*}\left(z_{n_{i}}\right) \rightarrow L^{*}(z)$ in ca $\left(\Sigma, E^{*}\right)$. Hence $L^{*}$ is compact. This proves (i).

To establish (ii) we make the following observations. The operator $L$ has dense range if and only if $L^{*}$ is a one to one mapping; hence $\delta(m)$ is a metric if and only if $L$ has dense range. Thus by (i), $L$ is compact with dense range if and only if $\left(F_{1}^{*}, \delta(m)\right)$ is a compact metric space; and by (i) the $w^{*}$-topology is stronger than the $\delta(m)$-topology if $L$ is compact. Consequently, the theorem is proved.

5. Properties of $s$-bounded measures. In this section we continue our investigation of strongly bounded representing measures. An example is given which illustrates the pathological behavior of operator valued representing measures. Following this example, three notions of absolute continuity are discussed and additional miscellaneous results are given. 
Theorem 5.1. The following conditions are equivalent:

(a) the $B$-space $F$ does not contain a topological isomorph of $c_{0}$;

(b) for each $E$ and each $H$, the limit of every pointwise convergent uniformly bounded (in semivariation) sequence of s-bounded representing measures $\mu^{(i)}$ : $\Sigma \rightarrow B(E, F)$ is an s-bounded representing measure;

(c) for each $E$ and each $H$, a representing measure $m$ is s-bounded if and only if $m: \Sigma \rightarrow B(E, F)$.

Proof. Since Example 5.1 infra and the remarks following it show that if $F \supset c_{0}$ then there is a representing measure $m$ which is countably additive, not $s$ bounded, and the pointwise limit of a uniformly bounded sequence of $s$-bounded representing measures, it suffices to show that (a) $\Rightarrow(b)$ and (a) $\Rightarrow(c)$.

(a) $\Rightarrow$ (c). Suppose that $F$ does not contain $c_{0}$ and $m: \Sigma \rightarrow B(E, F)$. Choose $\left(A_{i}\right)$ disjoint, $\left(x_{i}\right) \subset E_{1}$, and $z \in F^{*}$. Since $\sum\left|\left\langle m\left(A_{i}\right) x_{i}, z\right\rangle\right| \leq \sum\left|m_{z}\right|\left(A_{i}\right)<\infty$, $\sum m\left(A_{i}\right) \cdot x_{i}$ is weakly unconditionally convergent, and by a result of Bessaga and Pełczyński [4, p. 160], $\sum m\left(A_{i}\right) x_{i}$ is unconditionally convergent. Thus $m$ is $s-$ bounded by Lemma 3.1 .

Conversely, if $m$ is $s$-bounded, then $m$ is countably additive and $m: \Sigma$ $\rightarrow B(E, F)$ by Corollary 4.4.1.

(a) $\Rightarrow$ (b). Suppose that $c_{0} \oplus F$ and $\left(\mu^{i}\right)$ is a sequence satisfying the hypothesis of (b). Set $\mu(A)=\lim \mu^{i}(A), A \in \Sigma$. Since $\tilde{\mu}^{i}(H)$ is uniformly bounded, it is clear that $\tilde{\mu}(H)<\infty$. Therefore to complete the argument it is enough to show that $\mu$ is countably additive and $\left|\mu_{z}\right| \in \operatorname{rca}(\Sigma, \mathbb{C})$ for each $z \in F^{*}$. For we know that $\mu: \Sigma \rightarrow B(E, F)$, and, by the proof of the preceding implication, to see that $\mu$ is $s$-bounded it suffices to prove that $\mu$ is a representing measure. Since each $\mu^{i}$ is $s$-bounded, there is a $\lambda_{i} \geq 0, \lambda_{i} \in \operatorname{rca}(\Sigma, \mathbb{C})$, so that $\tilde{\mu}^{i}(A) \rightarrow 0$ as $\lambda_{i}(A) \rightarrow 0$ (see Dunford and Schwartz, [13, IV.13.20(iii)]). Let $\lambda=\sum_{i=1}^{\infty} \lambda_{i} / 2^{i}\left(\left|\lambda_{i}\right|(H)+1\right)$; note that $\mu^{i} \ll \lambda$. By the Nikodym theorem, $\mu$ is countably additive. Furthermore, by the Vitali-Hahn-Saks theorem, $\mu_{z}^{i} \ll \lambda$ uniformly in $i$ for each $z \in F^{*}$. Therefore $\mu_{z}: \Sigma \rightarrow E^{*}$ is regular for each $z$, and, by Corollary 2.8.1, $\left|\mu_{z}\right|$ $\in \mathrm{rca}(\Sigma)$. This completes the proof of the theorem.

Recall that an operator $Y$ from an arbitrary $B$-space $X$ into a $B$-space $Y$ is unconditionally convergent provided it maps weakly unconditionally convergent series in $X$ to unconditionally convergent series in $Y$. The Orlicz-Pettis theorem implies that every weakly compact operator $T: X \rightarrow Y$ is unconditionally convergent.

Theorem 5.2. If $c_{0} \llbracket E$, then every s-bounded operator $m \leftrightarrow L$ : $C_{0}(H, E) \rightarrow F$ is unconditionally convergent.

Proof. Let $\Sigma f_{i}$ be weakly unconditionally convergent in $C_{0}(H, E)$. Since $H \otimes E^{*} \subset B^{*}$, it follows that $\sum f_{i}(t)$ is weakly unconditionally convergent in $E$ for each $t \in H$. But, $E \triangleright c_{0}$, and $\sum f_{i}(t)$ is unconditionally convergent in $E$. Set $f_{0}(t)=\lim _{n} \sum_{i=1}^{n} f_{i}(t), t \in H$, and let $\delta_{n}(t)=\sum_{i=1}^{n} f_{i}(t), n=1,2, \ldots$ By the uniform boundedness principle, $\sup \left\{\left\|\delta_{n}\right\|_{\infty}\right\}<\infty$. 
Now since $m$ is $s$-bounded, $K=\left\{\left|m_{z}\right|: z \in F_{1}^{*}\right\}$ is conditionally weakly compact in $\mathrm{ca}(\Sigma, C)$; thus by $[13$, IV.9.1] there is a positive $\lambda \in \mathrm{ca}(\Sigma, \mathbb{C})$ so that $K \ll \lambda$ uniformly and $\lambda(A) \leq \sup \left\{\left|m_{z}\right|(A): z \in F_{1}^{*}\right\}$. Therefore $\lambda(A) \rightarrow 0$ if and only if $\tilde{m}(A) \rightarrow 0$, and $\lambda$ is a control measure for $m$ in the sense of Bartle [1]. By the bilinear dominated convergence theorem in [1] it follows that $\int f_{0} d m$ $=\lim _{n} \int \delta_{n} d m=\lim _{n} \sum_{i=1}^{n} L\left(f_{i}\right)$.

Finally, if $\left(n_{k}\right)$ is any permutation of the natural numbers, then $\sum_{k} f_{n_{k}}(t)$ $\rightarrow f_{0}(t), \quad t \in H$, since $\sum f_{i}(t)$ is unconditionally convergent. Therefore $\lim _{p} \sum_{k=1}^{p} L\left(f_{n_{k}}\right)=\int f_{0} d m$, and the theorem is proved.

The following example was mentioned in Theorem 5.1.

Example 5.1. Let $E_{n}$ be Euclidean $2^{n}+1$ dimensional space with the $l^{1}$-norm, and let $E=\left(\prod_{n=1}^{\infty} E_{n}\right)$, i.e. $E$ is the collection of all functions $f$ defined on the natural numbers so that $f(i) \in E_{i}$ and $\sup \{\|f(i)\|: i=1,2, \ldots\}<\infty$. Note that elements of $E$ may act on $E$ and transform it into $l^{\infty}$, i.e. if $\left(x_{n}\right),\left(y_{n}\right) \in E$, then $\left\langle\left(x_{n}\right),\left(y_{n}\right)\right\rangle=\left(z_{n}\right) \in l^{\infty}$, where $z_{n}$ is the inner product of $x_{n}$ and $y_{n}$. Let $H$ be a countably infinite discrete space whose elements are written in the form $\left\{\mathbb{Q}_{i, j(i)}: i=1,2, \ldots, 1 \leq j(i) \leq 2^{i}+1\right\}$, and let $A_{i}=\left\{\mathbb{Q}_{i, 1}, \ldots, \mathbb{Q}_{i, 2^{i}+1}\right\}$. Now define $m\left(\left\{\mathbb{Q}_{i, j}\right\}\right)$ to be that point in $E$ with $1 / 2^{i}$ in $j$ th component of the $i$ th coordinate and 0 elsewhere; define $m(\varnothing)$ to be 0 . Therefore $m\left(\left\{Q_{i, j}\right\}\right) \in E$ for each $i$ and $j$; in addition $m\left(\left\{Q_{i, j}\right\}\right): E \rightarrow c_{0}$ is an operator. If $A \in \Sigma$, define $m(A)$ to be the point in $E$ whose $j$ th component of the $i$ th coordinate is $m\left(A \cap\left\{Q_{i, j}\right\}\right)$. Then $m(A): E \rightarrow c_{0}$ is an operator for each $A \in \Sigma$. In fact, if $y=\left(y_{n}\right) \in E_{1}$, then $m\left(A_{i}\right) y=\left(z_{n}\right) \in c_{0}$ so that $t_{n}=0$ if $n \neq i$ and $\left.\left|z_{i}\right|=K\left(1 / 2^{i}, \ldots, 1 / 2^{i}\right), y_{i}\right\rangle \mid$ $\leq 1 / 2^{i}$. From this inequality, it follows easily that $m$ is countably additive. But, since $\tilde{m}\left(A_{i}\right)=\left(2^{i}+1\right) / 2^{i}, m$ is not $s$-bounded.

Remark 5.2. As we indicated in Theorem 5.1, this example answers two other questions dealing with vector measures. In [9], using Rickart's notion of sboundedness, Brooks and Jewett showed that the pointwise limit of a sequence of $s$-bounded set functions was $s$-bounded. This result fails for representing measures. In fact, if $\left(A_{i}\right)$ and $m$ are as in Example 5.1, then defining $m_{n}(A)$ to be $m\left(A \cap\left(\cup_{i=1}^{n} A_{i}\right)\right)$ and using the countable additivity of $m$, it follows that $m_{n}(A) \rightarrow m(A)$. In addition, it is not difficult to see that each $m_{n}$ is $s$-bounded.

Secondly, Batt and Berg [3] have given an example of a compact operator. $L: B \rightarrow F$ whose representing measure does not have conditionally compact range. Example 5.1 shows that the conditional compactness of range $(m)$ and the compactness (even nuclearity) of each $m(A)$ does not imply that the represented operator is even weakly compact. In fact, if $A \in \Sigma$, then $f_{n}=m\left(A \cap A_{n}\right)$ may be naturally interpreted as an element of $E^{*}$. As such, $\left\|f_{n}\right\| \leq 1 / 2^{n}$. Now let $e_{n}$ denote the $n$th unit vector in $l^{\infty}$, and note that $f_{n} \otimes e_{n}$ is an operator from $E$ to $c_{0}$, where $\otimes$ denotes tensor product, e.g., see Schaefer [30, pp. 97-100]. The countable additivity of $m$ then implies that

$$
m(A)=\sum_{n=1}^{\infty} \frac{1}{2^{n}}\left(2^{n} f_{n}\right) \otimes e_{n},
$$


where $\left\|2^{n} f_{n}\right\| \leq 1$ and $\left\|e_{n}\right\|=1$. But this is Grothendieck's characterization of nuclear operators [17].

Now let $K=\{m(A): A \in \Sigma\}$, and let $\varepsilon>0$. There is an $N$ so that if $A \in \Sigma(H), A \subset B=\cup A_{n}, n \geq N$, then $\|m(A)\|<\varepsilon$. But there are only finitely many sets in $\Sigma$ which do not meet $B$. Thus $K$ is totally bounded; consequently, $K$ is conditionally compact.

Next we define three types of absolute continuity for representing measures and briefly study permanence of compact and weakly compact operators with respect to these concepts of absolute continuity.

Definition 5.1. Let each of $n, m: \Sigma(H) \rightarrow B\left(E, F^{* *}\right)$ be a representing measure.

(i) We say that $n$ is weakly absolutely continuous with respect to $m(n<m)$ if for each $A \in \Sigma(H)$ and $x \in E$

$$
n(A) \cdot x \in \overline{\left\{\bigcup_{\pi(A)} \sum m\left(A_{i}\right) \cdot x_{i}: x \in E\right\}} .
$$

(ii) We say that $n$ is absolutely continuous with respect to $m(n \ll m)$ if for each $\varepsilon>0$ there is a $\delta>0$ so that if $\tilde{m}(A)<\delta$, then $\tilde{n}(A)<\varepsilon$.

(iii) We say that $n$ is strongly absolutely continuous with respect to $m(n \ll m)$ if for each $A \in \Sigma$ and $x \in E_{1}$ then

$$
n(A) \cdot x \in \overline{\left\{\bigcup_{\pi(A)} \sum m\left(A_{i}\right) \cdot x_{i}: x_{i} \in E_{1}\right\}} .
$$

Remarks 5.3. In Lewis [24] it was shown that $n<m$ if and only if $\left|n_{z}\right| \ll\left|m_{z}\right|$; in [23] it was shown that if $n \lll m$, then $\left|n_{z}\right| \leq\left|m_{z}\right|$ for each $z \in F^{*}$.

If $n \leftrightarrow T$ and $m \leftrightarrow L$, then we write $T<(\ll)(\lll) L$ to indicate $n<$ $(\ll)(\lll) m$. We are concerned with the following questions. If $T<(\ll)(\ll) L$, and $L$ is (weakly) compact, then must $T$ be (weakly) compact? We obtain positive answers in the following cases.

Theorem 5.3. (i) If $L$ is a compact operator and $T \lll L$ then $T$ is a compact operator.

(ii) If $E^{*}$ and $E^{* *}$ have property $R-N, L$ is weakly compact, and $T \lll L$, then $T$ is weakly compact.

(iii) If $E$ is reflexive, $L$ is weakly compact, and $\tilde{m}(A)=0 \Rightarrow \tilde{n}(A)=0$, then $T$ is weakly compact if and only if $n \ll m$.

Proof. (i) Since $L$ is compact, $\left(F_{1}^{*}, \delta(m)\right)$ is a compact pseudometric space. By Remark 5.3 above, $\left|n_{z}\right|(A) \leq\left|m_{z}\right|(A), z \in F^{*}, A \in \Sigma$. Therefore $\delta(n)(z) \leq$ $\delta(m)(z),\left(F_{1}^{*}, \delta(n)\right)$ is a compact space, and $T$ is a compact operator.

(ii) Let $\Gamma(A)=\left\{\cup_{\pi(A)} \sum m\left(A_{i}\right) x_{i}: x_{i} \in E_{1}\right\}$. Then, since $\Gamma(A) \subset L^{* *}\left(S_{E}(\Sigma)_{1}\right)$ and $L$ is weakly compact, $\Gamma(A)$ is conditionally weakly compact. Thus $\overline{\Gamma(A)}$ is conditionally weakly compact, and since $\left\{m(A) x: x \in E_{1}\right\} \subset \overline{\Gamma(A)}, m(A)$ is a weakly compact operator for each $A$. Therefore, by Remark 5.3, $n$ is s-bounded, and, by Theorem $4.1, T$ is weakly compact. 
(iii) Since $E$ is reflexive, we immediately see that $n(A)$ is weakly compact for each $A \in \Sigma$. And in Lewis [25, Theorem 3.1] it was shown that if $m$ is $s$-bounded and $\tilde{m}(A)=0 \Rightarrow \tilde{n}(A)=0$, then $n$ is s-bounded if and only if $n \ll m$. Therefore, by Theorem 4.1, (iii) follows, and the theorem is proved.

Our next theorem is an analog of Theorem 7, p. 158 of Dinculeanu [11], i.e. if $E$ is a $B$-space we obtain a measure theoretic identification of the extreme points of $\mathbf{B}_{1}^{*}$. Denote this set by $\operatorname{ext}\left(\mathbf{B}_{1}^{*}\right)$. Clearly we cannot conclude that an extreme point will be multiplicative in our setting.

Theorem 5.4. If $L \in \operatorname{ext}\left(\mathrm{B}_{1}^{*}\right)$ and $L \leftrightarrow m^{*}$ then $\operatorname{supp}(m)$ is a singleton.

Proof. If $A_{1}$ and $A_{2}$ are disjoint members of $\Sigma$, we first show that

$$
m\left(A_{1}\right)=0 \text { or } m\left(A_{2}\right)=0 .
$$

For, if not, let $m_{1}(A)=m\left(A \cap A_{1}\right)$ and $m_{2}(A)=m\left(A-A_{1}\right), A \in \Sigma$. Then

$$
\begin{aligned}
\tilde{m}(H) & =|m|(H)=\tilde{m}_{1}(A)+\tilde{m}_{2}\left(H-A_{1}\right) \\
& =\left|m_{1}\right|\left(A_{1}\right)+\left|m_{2}\right|(H-A)=1,
\end{aligned}
$$

since the semivariation and total variation are the same in this case. Now let $n_{1}=m_{1} /\left(\left|m_{1}\right|(H)\right)$, and let $n_{2}=m_{2} /\left(\left|m_{2}\right|(H)\right)$. Then $n_{1} \neq m, n_{2} \neq m$, and $\left|m_{1}\right|(H) n_{1}+\left|m_{2}\right|(H) n_{2}=m$. Therefore $L$ cannot be extreme, and we have a contradiction. Thus if $A_{1} \cap A_{2}=\varnothing$, then $m\left(A_{1}\right)=0$ or $m\left(A_{2}\right)=0$, and the only values assumed by $m$ are 0 and $m(H)$. Therefore, in particular, $m(A)=0$ if and only if $\tilde{m}(A)=0$.

Now if $x$ and $y$ are distinct points in $\operatorname{supp}(m)$, let $U_{x}$ and $U_{y}$ be disjoint open sets containing $x$ and $y$ respectively. But then $m\left(U_{x}\right)=0$ or $m\left(U_{y}\right)=0$, and $\tilde{m}\left(U_{x}\right)=0$ or $\tilde{m}\left(U_{y}\right)=0$. Consequently, either $x$ or $y$ does not belong to $\operatorname{supp}(m)$, a contradiction, and the proof is finished.

We remark that establishing (i) is the key to the proof of Theorem 7 in [11]. Furthermore, the converse of this theorem is false in this setting.

Example 5.2. Suppose that $H$ is a nontrivial compact Hausdorff space $E=l^{1}$, and $\mathbf{B}=C(H, E)$. Let $t \in H$, and, if $\left(x_{n}\right) \in l^{\infty}$, then define

$$
\left(\left(x_{n}\right), t\right)(f) \equiv\left\langle\left(x_{n}\right), f(t)\right\rangle, \quad f \in \mathbf{B} .
$$

Then certainly $\left(\left(x_{n}\right), t\right) \in \mathrm{B}^{*}$, and $\left\|\left(\left(x_{n}\right), t\right)\right\| \leq\left\|\left(x_{n}\right)\right\|$. Then let $m(A)=(1$, $0, \ldots, 0, \ldots)$ if $t \in A$ and 0 otherwise. Therefore $\tilde{m}(H)=|m|(H)=1$. But $m=1 / 2 m_{1}+1 / 2 m_{2}$, where $m_{1}(A)=(1,1,0, \ldots, 0, \ldots)$ if $t \in A$ and 0 otherwise and $m_{2}(A)=(1,-1,0, \ldots, 0, \ldots)$ if $t \in A$ and 0 otherwise. Since $\left|m_{1}\right|(H)$ $=\left|m_{2}\right|(H)=1$, it follows that $L(\cdot)=\int_{H}(\cdot) d m=(m(t), t)$ is not extreme.

We next state two theorems obtained in Lewis [26] - one providing us with a generalization of a result due to Grothendieck [17] and Bartle, Dunford, and 
Schwartz [2]-the other providing us with a partial result which raises an interesting question.

Theorem 5.5. Suppose E satisfies the Schur condition (weak and norm convergence of sequences are the same), and let $L: B \rightarrow F$ be an s-bounded operator, with representing measure $m$. Then

(i) $m: \Sigma \rightarrow B(E, F)$;

(ii) if $\left(f_{n}\right)$ is $\mathbf{B}^{*}$-Cauchy in $U_{E}(\Sigma)$, then $\left(L\left(f_{n}\right)\right)$ converges in $F$;

(iii) if $f_{n} \rightarrow \mathbf{B}^{*} f$ in $U_{E}(\Sigma)$, then $L\left(f_{n}\right) \rightarrow L(f)$.

Conversely, if $E$ is any $B$-space and $m$ is a representing measure which satisfies (i), (ii), and (iii), then $m$ is s-bounded.

Theorem 5.6. If $m$ is a representing measure, $m \leftrightarrow L$, then $m$ is $s$-bounded if and only if for each sequence of sets $A_{i} \searrow \varnothing$, there is a nested sequence $\left(U_{n}\right)$ of open sets so that $A_{n} \subset U_{n}$ and $L\left(f_{n}\right) \rightarrow 0$ uniformly for each sequence $\left(f_{n}\right)$ so that $\operatorname{supp}\left(f_{n}\right)$ $\subset U_{n},\left\|f_{n}\right\| \leq 1$.

Theorem 5.7. If $E$ satisfies the Schur condition, then no infinite dimensional reflexive subspace of $C_{0}(H, E)$ is complemented in $C_{0}(H, E)$.

Proof. Suppose $X$ is an infinite dimensional reflexive subspace of $C_{0}(H, E)$ and $P: C_{0}(H, E) \rightarrow X$ is a continuous projection. Since $X$ is reflexive, $P$ is weakly compact, and, by Theorem 5.5, $P^{2}=P$ is compact. But then $X_{1}$ must be compact in the norm topology, and this is a contradiction since $X$ is infinite dimensional.

In [2] and [17] this result was established for $E$ being the scalar field.

We conclude with the following two problems.

Problem 1. Using Theorem 5.6, it is not difficult to see that if $F=\mathbf{B}$, then $\mathscr{B}$ (= set of $s$-bounded operators) is a closed left ideal. Is $\mathscr{B}$ also a right ideal?

Problem 2. Give a measure theoretic characterization of the extreme points (provided any exist) of the closed unit ball of $B(B, F)$.

\section{REFERENCES}

1. R. G. Bartle, A general bilinear vector integral, Studia Math. 15 (1956), 337-352. MR 18, 289.

2. R. G. Bartle, N. Dunford and J. Schwartz, Weak compactness and vector measures, Canad. J. Math. 7 (1955), 289-305. MR 16, 1123.

3. J. Batt and E. J. Berg, Linear bounded transformations on the space of continuous functions, J. Functional Analysis 4 (1969), 215-239. MR 40 \# 1798.

4. C. Bessaga and A. Pelczyński, On bases and unconditional convergence of series in Banach spaces, Studia Math. 17 (1958), 151-164. MR 22 \#5872.

5. J. K. Brooks, On the existence of a control measure for strongly bounded vector measures, Bull. Amer. Math. Soc. 77 (1971), 999-1001. MR 44 \#4178.

6. Weak compactness in the space of vector measures, Bull. Amer. Math. Soc. 78 (1972), $284-287$.

7. Contributions to the theory of finitely additive measures, Advances in Math. (to appear).

8. Equicontinuous sets of measures and applications to Vitali's integral convergence theorem and control measures, Advances in Math. 10 (1973), 165-171. 
9. J. K. Brooks and R. S. Jewett, On finitely additive vector measures, Proc. Nat. Acad. Sci. U.S.A. 67 (1970), 1294-1298. MR 42 \#4697.

10. J. K. Brooks and P. W. Lewis, Operators on function spaces, Bull. Amer. Math. Soc. 78 (1972), 697-701.

11. N. Dinculeanu, Vector measures, Internat. Series of Monographs in Pure and Appl. Math., vol. 95, Pergamon Press, New York; VEB Deutscher Verlag der Wissenschaften; Berlin, 1967. MR 34 \#6011b.

12. N. Dunford and B. J. Pettis, Linear operations on summable functions, Trans. Amer. Math. Soc. 47 (1940), 323-392. MR 1, 338.

13. N. Dunford and J. T. Schwartz, Linear operators. I. General theory, Pure and Appl. Math., vol. 7, Interscience, New York, 1958. MR 22 \#8302.

14. J. R. Edwards and S. G. Wayment, A unifying representation theorem, Math. Ann. 187 (1970), 317-328. MR 42 \# 5074.

15. C. Foiaş and I. Singer, Some remarks on the representation of linear operators in spaces of vector valued continuous functions, Rev. Math. Pures Appl. 5 (1960), 729-752. MR 24 \#A1618.

16. R. K. Goodrich, A Riesz representation theorem, Proc. Amer. Math. Soc. 24 (1970), $629-636$.

17. A. Grothendieck, Sur les applications linéaires faiblement compactes d'espaces du type $C(K)$, Canad. J. Math. 5 (1953), 129-173. MR 15, 438.

18. L. Gillman and M. Jerison, Rings of continuous functions, University Series in Higher Math., Van Nostrand, Princeton, N.J., 1960. MR 22 \#6994.

19. P. R. Halmos, Measure theory, Van Nostrand, Princeton, N.J., 1950. MR 11, 504.

20. L. Hormander, Linear partial differential operators, Die Grundlehren der math. Wissenschaften, Band 116, Academic Press, New York; Springer-Verlag, Berlin, 1963. MR 28 \#4221.

21. P. W. Lewis, Extension of operator valued set functions with finite semivariation, Proc. Amer. Math. Soc. 22 (1969), 563-569. MR 39 \#7061.

22. - Some regularity conditions on vector measures with finite semivariation, Rev. Roumaine Math. Pures Appl. 15 (1970), 375-384. MR 41 \#8626.

23.—, Vector measures and topology, Rev. Roumaine Math. Pures Appl. 16 (1971), 1201-1209.

24._, Addendum to: Vector measures and topology, Rev. Roumaine Math. Pures Appl. 16 (1971), 1211-1213.

25.—, Regularity conditions and absolute continuity for vector measures, J. Reine Angew. Math. 247 (1971), 80-86. MR 43 \#4997.

26._- Variational semiregularity and norm convergence, J. Reine Angew. Math. (to appear).

27. R. S. Phillips, On weakly compact subsets of a Banach space, Amer. J. Math. 65 (1943), 108-136. MR 4, 218.

28. C. E. Rickart, Decompositions of additive set functions, Duke Math. J. 10 (1943), 653-665. MR $5,232$.

29. W. Rudin, Real and complex analysis, McGraw-Hill, New York, 1966. MR 35 \# 1420.

30. H. H. Schaefer, Topological vector spaces, Macmillan, New York, 1966. MR 33 \#1689.

31. I. Singer, Sur les applications linéaires intégrales des espaces de fonctions continues. I, Rev. Math. Pures Appl. 4 (1959), 391-401. MR 22 \#5883.

32. K. Swong, A representation theory of continuous linear maps, Math. Ann. 155 (1964), 270-291; errata, ibid. 157 (1964), 178. MR 29 \#2642.

33. E. O. Thorp and R. J. Whitley, Operator representation theorems, Illinois J. Math. 9 (1965), 595-601. MR 31 \#6126.

34. D. H. Tucker, A representation theorem for a continuous linear transformation on a space of continuous functions, Proc. Amer. Math. Soc. 16 (1965), 946-953. MR 33 \#7865.

Department of Mathematics, University of Florida, Gainesville, Florida 32601

Department of Mathematics, North Texas State University, Denton, Texas 76203 